\title{
Pricing electricity derivatives within a Markov regime-switching model: a risk premium approach
}

\author{
Joanna Janczura
}

Received: 8 March 2013 / Accepted: 6 August 2013 / Published online: 25 August 2013

(C) The Author(s) 2013. This article is published with open access at Springerlink.com

\begin{abstract}
In this paper we derive analytic formulas for electricity derivatives under assumption that electricity spot prices follow a 3-regime Markov regime-switching model with independent spikes and drops and periodic transition matrix. Since the classical derivatives pricing methodology cannot be used in the case of non-storable commodities, we employ the concept of the risk premium. The obtained theoretical results are then used for the European Energy Exchange data analysis. We calculate the risk premium in the case of the calibrated 3-regime MRS model. We find a time varying structure of the risk premium and an evidence for a negative risk premium (or positive forward premium), especially at short times before delivery. Finally, we use the obtained risk premium to calculate prices of European options written on spot, as well as, forward prices.
\end{abstract}

Keywords Regime-switching model - Electricity spot price - Derivatives pricing · Risk premium

\section{Introduction}

Deregulation of electricity markets has led to a substantial increase in risk borne by market participants. The often unexpected extreme spot price changes caused by nonstorable nature of electricity range even two orders of magnitude and can cause severe financial problems to the utilities that buy electricity in the wholesale market and deliver it to consumers at fixed prices. The utilities and other power market companies need to hedge against this price risk. A straightforward way to do it is to use derivatives,

J. Janczura $(\bowtie)$

Hugo Steinhaus Center, Institute of Mathematics and Computer Science, Wrocław University of Technology, 50-370 Wrocław, Poland

e-mail: joanna.janczura@pwr.wroc.pl 
like forwards and options. It is exactly the aim of this paper to price commonly traded electricity derivatives.

Before calculating a price of a derivative, a proper model for the underlying asset has to be chosen. There are two approaches common for the electricity market. The first one is to start with specifying the forward price dynamics (see e.g. Clewlow and Strickland 1999; Benth and Koekebakker 2008; Bjerksund et al. 2010). Such approach is useful if only derivatives written on forwards are to be considered and a link between forward and spot prices is not important for modeling issues. Obviously, the spot price can always be derived using the fact that the forward and spot prices should coincide at the forward settlement. However, the complexity of the spot price dynamics is then usually neglected. The second approach is based on defining the spot price dynamics first (see e.g. Lucia and Schwartz 2002; Miltersen 2003; Benth et al. 2003; Bierbrauer and Menn 2007) and then modeling a link between the forward and spot markets. Usually, a convenience yield or risk premium notion is used (Benth et al. 2008a; Geman 2005; Weron 2006; Benth and Sgarra 2012; Haerdle and LopezCabrera 2012; Longstaff and Wang 2004; Lucia and Torro 2008; Benth et al. 2008b; Benth and Meyer-Brandis 2009). Using such an approach allows to price derivatives written both on the spot and the forward price. Moreover, a relation between spot and forward prices is taken into account and the lack of forward price data is no more a limitation. Here, we use the latter approach and describe the spot price dynamics by a Markov regime-switching (MRS) model with independent spikes and drops and periodic transition matrix that was proposed by Janczura and Weron (2010). For other applications of MRS models to electricity prices see e.g. Deng (1998), Ethier and Mount (1998), Huisman and Jong (2003), Huisman and Mahieu (2003), Kholodnyi (2005), De Jong (2006), Haldrup and Nielsen (2006), Mount et al. (2006), Becker et al. (2007), Huisman (2008), Kanamura and Ohashi (2008), Karakatsani and Bunn (2008), Mari (2008), Weron (2009), Erlwein et al. (2010), Haldrup et al. (2010), Dempster et al. (2013).

After specifying a model we have to choose derivatives pricing methodology. Classical approach used in financial and commodities markets is based on the no-arbitrage assumption and construction of a strategy replicating a future payoff (or equivalently finding a martingale measure, Musiela and Rutkowski 1997). However, such approach fails in case of electricity due to very limited storage possibilities. Therefore, instead of using a martingale approach, we employ a concept of the risk premium/market price of risk and find such pricing measure that yields the observed forward market prices. With such methodology we are able to derive forward prices from the spot price model that coincide with the actual quotations and also to find explicit formulas for premiums of European options written on spot, as well as, on forward prices within the assumed 3-regime MRS model. The theoretical results are then used for the European Energy Exchange (EEX) data analysis. We calculate the risk premium and find a time varying structure of the risk premium, which increases with increasing time to delivery. Moreover, we obtain a strong evidence for a negative risk premium (or positive forward premium) in the EEX market, especially at short times before delivery.

The paper is structured as follows. In Sect. 2 we introduce the Markov regimeswitching model used for electricity spot price dynamics. Next, in Sect. 3 we explain 
why the classical derivatives pricing approach fails in case of electricity derivatives and, as a solution, we describe the 'risk premium' approach in case of the considered model. The obtained results are then used to derive analytical formulas for prices of electricity derivatives in Sect. 4. Finally, in Sect. 5 we use the obtained theoretical results to price derivatives using the European Energy Exchange (EEX) data and in Sect. 6 we conclude. All of the computations in the empirical part of the paper were conducted in Matlab 2009.

\section{The model}

Let us first recall the main stylized facts about electricity prices. Electricity prices highly depend on the actual demand/consumption. Obviously, the latter is varying during a year due to the changing weather conditions and throughout the week or day due to the business cycle. The same long-term (yearly) and short-term (weekly/daily) seasonality is recorded for electricity prices. The second apparent feature is the very high volatility of electricity prices and unexpected, usually transient, dramatic price changes called spikes or jumps. The spot price may rise for a few hours to even two orders of magnitude of the standard prices and then fall back to the normal level. What makes electricity market completely different from other financial or commodities markets is that the electricity prices may, as well, abruptly fall down yielding negative values. Finally, electricity prices are mean-reverting, meaning that in long time period they move back to some equilibrium level.

Seasonality is usually removed from the analyzed prices prior to modeling by fitting some periodic function like sine (Cartea and Figueroa 2005; De Jong 2006) or piecewise constants (Lucia and Schwartz 2002). Alternatively, some smoothing technique like wavelets or moving average can be used (Weron 2009; Nowotarski et al. 2013). The mean-reverting property is typically modeled with some mean-reverting processes, like e.g. AR(1) time series or the Vasicek (1977) model. The most challenging for modeling, and at the same time the most important for risk management, are the price spikes. One approach is to incorporate a jump component into a standard mean-reverting diffusion model (Deng 1998; Cartea and Figueroa 2005; Weron 2008). However, in the resulting jump-diffusion models there is a problem of how the price after a spike revert back to the normal level. Nor an immediate negative jump, nor mean-reversion pulling back the prices to the normal level, yields a flexible tool for modeling consecutive spikes. Another possibility are the Markov regime-switching (MRS) models in which the prices might stay in the excited (spike) regime with some probability. Hence, MRS models allow for modeling consecutive spikes in a very natural way and seem to be a reasonable choice for electricity price dynamics. To our best knowledge MRS models were first applied to electricity prices by Ethier and Mount (1998) who used an AR(1) time series with parameters depending on the actual regime. A MRS model with independent spikes was later introduced by Huisman and Jong (2003) and De Jong (2006). Numerous attempts improving statistical properties of the model (see e.g. Huisman and Mahieu 2003; Haldrup and Nielsen 2006; Weron 2009; Dempster et al. 2013) or including some exogenous factors (see e.g. Huisman 2008; Kanamura and Ohashi 2008; Karakatsani and Bunn 2008) were later proposed. Here, we focus on a 3-regime model with independent spikes and drops introduced recently 
by Janczura and Weron (2010), who found it superior to other MRS models. It allows for modeling consecutive spikes and drops as independent variables, being flexible enough to capture different statistical properties of spikes and drops, which are a consequence of different driving factors. Moreover, it takes into account the seasonal intensity of the occurrences of extreme observations. Finally, the model not only reproduces the stylized facts about electricity prices, but also it's goodness of fit was statistically confirmed for different markets, see Janczura and Weron (2010) for more details, and is also confirmed for the data studied in this paper.

Having in mind the above mentioned features of electricity, we let the electricity spot price be given by

$$
P_{t}=g_{t}+X_{t}
$$

where $g_{t}$ is a deterministic seasonal component and $X_{t}$ follows a 3-regime MRS model with independent spikes and drops. Namely,

$$
X_{t}=\left\{\begin{array}{lll}
X_{t, b} & \text { if } & R_{\lfloor t\rfloor}=b, \\
X_{\lfloor t\rfloor, s} & \text { if } & R_{\lfloor t\rfloor}=s, \\
X_{\lfloor t\rfloor, d} & \text { if } & R_{\lfloor t\rfloor}=d,
\end{array}\right.
$$

where $b$ denotes the base regime (describing the 'normal' prices), $s$ the spike regime (representing the sudden upward price jumps), while $d$ stands for the drop regime (responsible for the sudden price drops). Further, $\lfloor t\rfloor$ denotes the integer part of $t, R_{k}$ is a discrete-time Markov chain defined by a time-varying (periodic) transition matrix $\mathbf{P}(k)$

$$
\mathbf{P}(k)=\left(p_{i j}(k)\right)=\left(\begin{array}{lll}
p_{b b}(k) & p_{b s}(k) & p_{b d}(k) \\
p_{s b}(k) & p_{s s}(k) & p_{s d}(k) \\
p_{d b}(k) & p_{d s}(k) & p_{d d}(k)
\end{array}\right)
$$

where $p_{i j}(k)$ is the probability of switching from state $i$ at time $k$ to state $j$ at time $k+1$ for $k \in\{0,1,2, \ldots\}$. The process $R_{t}$ describes the actual state of the market, i.e. normal (base regime, denoted by ' $b$ ') behavior, spike (denoted by 's') or drop (denoted by ' $d$ '), and is an unobserved random variable. The base regime dynamics is given by the Vasicek (1977) model:

$$
d X_{t, b}=\left(\alpha-\beta X_{t, b}\right) d t+\sigma_{b} d W_{t},
$$

having unique mean-reverting solution of the form:

$$
X_{t, b}=X_{0, b} e^{-\beta t}+\frac{\alpha}{\beta}\left(1-e^{-\beta t}\right)+\sigma_{b} \int_{0}^{t} e^{-\beta(t-u)} d W_{u},
$$

where $W_{t}$ is a Wiener process (or Brownian motion), $\beta$ is the speed of mean-reversion, $\frac{\alpha}{\beta}$ is the long-time equilibrium level and $\sigma_{b}$ is the volatility. Note that, for simplicity, 
we use a constant volatility for the base regime. Assuming time varying $\sigma_{b}$ would make the estimation of the MRS model computationally intractable and might also lead to overparametrization. However, with the assumed model specification, the shifts in volatility observed in electricity prices can still be modeled by switching between regimes having different volatilities. The spike regime values $\left(X_{0, s}, X_{1, s}, X_{2, s}, \ldots\right)$ constitute an i.i.d. sample from the $c_{s}$-shifted log-normal distribution, i.e. :

$$
\ln \left(X_{k, s}-c_{s}\right) \sim N\left(\mu_{s}, \sigma_{s}^{2}\right), \quad X_{k, s}>c_{s},
$$

while the drop regime values $\left(X_{0, d}, X_{1, d}, X_{2, d}, \ldots\right)$ form an i.i.d. sample from the inverted $c_{d}$-shifted log-normal distribution defined as:

$$
\ln \left(-X_{k, d}+c_{d}\right) \sim N\left(\mu_{d}, \sigma_{d}^{2}\right), \quad X_{k, d}<c_{d}
$$

The log-normal distribution seems to be a good choice for modeling extreme observations like spikes or drops, as it has heavier tails than the Gaussian one, but still all moments are finite. Moreover the statistical goodness of fit of such distribution choice is confirmed in the empirical part of the paper, see Table 3. Obviously, for different data other distribution choice might be relevant. However, in such a case all of the following results can be easily adapted.

Observe, that in the model defined by (2) the price process can jump to a different regime only at discrete time points $t=0,1,2, \ldots$. This is motivated by the fact, that, even though the price is a result of continuous bidding, electricity spot price is typically settled for contracts with some delivery period, usually an hour. Hence, the change in electricity spot price dynamics may occur only in discrete time points.

Finally, let $(\Omega, \mathcal{F}, Q)$ be a probability space with filtration $\mathcal{F}_{t}$ generated by the processes $X_{t}$ and $R_{\lfloor t\rfloor}$ and assume a constant continuously compounded interest rate $r$. It should be noted that the market interest rates are seldom constant over a period of time. In the literature there are different approaches for dealing with this issue like a deterministic interest rate being a function of time or stochastic interest rate models ( see e.g. Vasicek 1977 or Heath et al. 1992). However, finding a deterministic function that will accurately describe future interest rates behavior is usually problematic, while the more advanced approaches often make derivatives pricing analytically intractable. On the other hand, interest rates in stable economies are not very volatile, especially over short periods of time, for example a change in a yearly LIBOR rate during the pricing period considered in the empirical example of Sect. 5 was only $0.06 \%$. Hence, for simplicity, we assume a constant interest rate.

\section{The risk premium}

The classical option pricing approach is based on the no-arbitrage assumption, which implies that the fair price of a derivative is the discounted expected future payoff under a martingale measure. If the market is complete, i.e. any contingent claim can be replicated with a self-financing strategy (it is attainable), there exists a unique martingale measure. However, due to non-storability of electricity a derivative written 
on the spot electricity price cannot be replicated with a portfolio consisting of the underlying instrument and a financing debt account. As a consequence, the market is incomplete. Moreover, since electricity cannot be traded in the usual way (once purchased has to be consumed), the only tradable asset in the spot market is the bank account (Benth et al. 2003). Recall that here we assume a continuously compounded constant interest rate $r$. Obviously, the discounted value of the bank account is a martingale under any measure equivalent to the actual (also called the objective or statistical) measure $Q$. Therefore, the spot market is arbitrage-free but there is no unique martingale measure. An additional criterion has to be used in order to select a pricing measure.

Here we use an approach based on the concept of the risk premium (see e.g. Benth and Sgarra 2012; Benth et al. 2008a; Geman 2005; Weron 2006), which is defined as a reward for investing into a risky asset instead of a risk-free one. In such methodology a risk-free asset is usually a derivative, while a risky asset is an underlying instrument. In the paper we consider a risk-free forward electricity contract and risky electricity spot price, i.e. the risk premium $R P$ is defined as the difference between the expected spot price in the future time $T$ and the price of a forward contract with delivery at time $T$

$$
R P(T)=E\left(P_{T} \mid \mathcal{F}_{0}\right)-f_{0}^{T},
$$

where $f_{0}^{T}$ is the market price at time 0 (now-the moment of pricing) of a forward contract expiring at time $T$. In such a case, the forward contract does not bring risk to it's owner, since the whole specification is agreed on the moment of entering a contract and, compared to the spot price, does not depend on unknown future market quotations.

Observe that, the definition (8) is based on the forward looking approach, as the risk premium is calculated using the forecast of the spot price. The risk premium defined in such a way is called in the literature (see e.g. Lucia and Torro 2008; Benth et al. 2008b; Karakatsani and Bunn 2005) the ex-ante risk premium. On the other hand, the risk premium is also often defined using the ex-post (historical) approach and calculated as the difference between the forward price and the realized spot price at the time of the forward contract delivery (see e.g. Geman and Vasciek 2001; Longstaff and Wang 2004). However, for the purpose of the paper we use the ex-ante risk premium. This is motivated by the fact, that for the valuation of a derivative with some future maturity time, the estimate of the future risk premium, rather than the realized one, should be used.

It should be noted that some authors (see e.g. Eydeland and Wolyniec 2003) define the risk premium as the forward premium, i.e. as the difference between the forward price and the expected spot price, being equal to $-R P(T)$. Moreover, in the classical financial and commodities setup there exists a notion of the convenience yield, which relates the current prices of forward contracts with the current spot prices using the storage theory (first introduced by Kaldor 1939), i.e. $f_{0}^{T}=P_{0} e^{(r-y) T}$, where $y$ is called the convenience yield. For commodities this relation is a consequence of the cost of carry relationship which means that the forward price must exceed the spot price by the cost of carrying the physical commodity, determined by the storage costs 
and the interest lost, until the expiry date of the contract (Weron 2006). However, in the case of non-storable commodities, like electricity, the storage theory rarely makes sense and the link between the current spot price and the forward prices is much more complex. Hence, instead we use the risk premium notion.

The idea of the 'risk premium' approach in derivatives valuation is to choose a martingale measure that is consistent with the prices of forward contracts quoted in the market. A similar approach is used in the weather (Benth and Benth 2007; Haerdle and Lopez-Cabrera 2012) or interest rate derivatives context and is based on calibrating the model to the initial yield curve (see e.g. Hull and White 1993; Bjork 1997).

Recall, that the arbitrage-free price of a forward contract should be equal to the expected future spot price under a pricing measure, namely

$$
f_{0}^{T}=E^{\lambda}\left(P_{T} \mid \mathcal{F}_{0}\right)
$$

where $f_{0}^{T}$ is the price at time 0 (now) of a forward contract with a delivery at time $T, P_{T}$ is the electricity spot price and $E^{\lambda}(\cdot)$ is the expected value with respect to the pricing probability measure $Q^{\lambda}$, equivalent to the actual measure $Q$. In an incomplete market relation (9) does not yield a unique forward price, as it is dependent on the choice of $Q^{\lambda}$. Here we choose $Q^{\lambda}$ such that relation (9) is consistent with market data, i.e. $E^{\lambda}\left(P_{T} \mid \mathcal{F}_{0}\right)$ is calibrated to the quotations of forward contracts. In other words, we choose the pricing measure that is used by the market. In the following we will assume that the measure $Q^{\lambda}$ is the probability measure under which the drift of the base regime process is parametrized by a function $\lambda(T)$ chosen so that $E^{\lambda}\left(P_{T} \mid \mathcal{F}_{0}\right)$ yields the market forward price $f_{0}^{T}$. The function $\lambda(T)$ is called the market price of risk, which can be seen as a drift adjustment in the dynamics of an asset to reflect how investors are compensated for bearing risk when holding the asset (Benth et al. 2008b).

Before we find the measure $Q^{\lambda}$, we give a brief explanation of how to calculate the risk premium in the 3-regime model (1)-(7). Assume, that the forward price $f_{0}^{T}$ is given for any maturity $T$ and the spot price model parameters $\theta=$ $\left(\alpha, \beta, \sigma_{b}, \mu_{s}, \sigma_{s}, c_{s}, \mu_{d}, \sigma_{d}, c_{d}, \mathbf{P}\right)$ are known.

Let $p_{i j}^{(t)}=P\left(R_{\lfloor t\rfloor}=j \mid R_{0}=i\right)$ denote the probability of switching from state $i$ at time 0 to state $j$ at time $\lfloor t\rfloor$. For a constant probability matrix $\mathbf{P}$ it is given by the $i j$ th element of the $\lfloor t\rfloor$ th power of the transition matrix, i.e. $p_{i j}^{(t)}=\left(\mathbf{P}^{\lfloor t\rfloor}\right)_{i j}$. For a time-varying probability matrix it is given by $p_{i j}^{(t)}=\left(\prod_{k=0}^{\lfloor t\rfloor} \mathbf{P}(k)\right)_{i j}$.

In order to simplify the derivation, in the following we assume that $P\left(R_{0}=b\right)=1$ or equivalently $X_{0}=X_{0, b}$ a.s., i.e. at time 0 the process $X_{t}$ is in the base regime with probability 1 .

Now, we can derive a formula for the risk premium. Observe that

$$
\begin{aligned}
E\left(X_{T} \mid \mathcal{F}_{0}\right)= & P\left(R_{\lfloor T\rfloor}=b \mid R_{0}=b\right) E\left(X_{T, b} \mid \mathcal{F}_{0}\right)+P\left(R_{\lfloor T\rfloor}=s \mid R_{0}=b\right) E\left(X_{\lfloor T\rfloor, s} \mid \mathcal{F}_{0}\right) \\
& +P\left(R_{\lfloor T\rfloor}=d \mid R_{0}=b\right) E\left(X_{\lfloor T\rfloor, d} \mid \mathcal{F}_{0}\right) .
\end{aligned}
$$

Recall, that $X_{\lfloor T\rfloor, s}$ and $X_{\lfloor T\rfloor, d}$ are random variables independent of $\mathcal{F}_{0}$. Hence, $E\left(X_{\lfloor T\rfloor, j} \mid \mathcal{F}_{0}\right)=E\left(X_{\lfloor T\rfloor, j}\right)$ for $j=s, d$. Moreover, from the assumption of $X_{0}=$ 
$X_{0, b}$ we have that $E\left(X_{T, b} \mid \mathcal{F}_{0}\right)=E\left(X_{T, b} \mid X_{0, b}\right)$. Hence,

$$
E\left(X_{T} \mid \mathcal{F}_{0}\right)=p_{b b}^{(T)} E\left(X_{T, b} \mid X_{0, b}\right)+p_{b s}^{(T)} E\left(X_{\lfloor T\rfloor, s}\right)+p_{b d}^{(T)} E\left(X_{\lfloor T\rfloor, d}\right) .
$$

As a consequence, from (1), (5) and (11), the risk premium in the 3-regime MRS model defined by Eqs. (2)-(7) is given by:

$$
\begin{aligned}
R P(T)= & p_{b b}^{(T)}\left[x_{0} e^{-\beta T}+\frac{\alpha}{\beta}\left(1-e^{-\beta T}\right)\right]+p_{b s}^{(T)}\left(e^{\mu_{s}+\frac{1}{2} \sigma_{s}^{2}}+c_{s}\right) \\
& +p_{b d}^{(T)}\left(-e^{\mu_{d}+\frac{1}{2} \sigma_{d}^{2}}+c_{d}\right)+g_{T}-f_{0}^{T}
\end{aligned}
$$

where $x_{0}$ is the stochastic part of the price observed at time 0 and $f_{0}^{T}$ is the market forward price.

Remark 1 Observe that, if assumption that $X_{0}=X_{0, b}$ is not satisfied we have:

$$
\begin{aligned}
E\left(X_{T, b} \mid \mathcal{F}_{0}\right)= & \mathbb{I}_{\left\{R_{0}=b\right\}} E\left(X_{T, b} \mid X_{0, b}\right) \\
& +\sum_{k=1}^{\infty} \mathbb{I}_{\left\{R_{0} \neq b, R_{-1} \neq b, R_{-2} \neq b, \ldots, R_{-k+1} \neq b, R_{-k}=b\right\}} E\left(X_{T, b} \mid X_{-k+1, b}\right),
\end{aligned}
$$

where a negative time index is used for the historical (i.e. before the moment of valuation $t=0)$ values of the process and for $u<T E\left(X_{T, b} \mid X_{u, b}\right)=X_{u, b} e^{-\beta(T-u)}+$ $\frac{\alpha}{\beta}\left(1-e^{-\beta(T-u)}\right)$. Note, that formula (13) is a consequence of the fact that the base regime values become latent if a spike or drop occurs. Moreover,

$$
P\left(R_{\lfloor T\rfloor}=j \mid \mathcal{F}_{0}\right)=\sum_{i \in\{b, s, d\}} \mathbb{I}_{\left\{R_{0}=i\right\}} P\left(R_{\lfloor T\rfloor}=j \mid R_{0}=i\right)=\sum_{i \in\{b, s, d\}} \mathbb{I}_{\left\{R_{0}=i\right\}} p_{i j}^{(T)} .
$$

Thus, the risk premium calculation and all of the following results can be generalized to the case $R_{0} \neq b$.

\section{Electricity derivatives pricing}

\subsection{Options written on the electricity spot price}

Now, we turn to pricing of a European call option written on the electricity spot price. Recall, that the European option is a contract that gives the buyer the right to buy/sell the underlying commodity at some future date $t$ (called maturity) at a certain price $K$ (called the strike price). First, we find the pricing measure $Q^{\lambda}$. Like Merton (1976) in the context of jump-diffusion processes we assume that the dynamics of spikes and drops are the same in the actual and pricing measures. Similar simplification was used e.g. by De Jong and Huisman (2002) or Weron (2008). It allows to avoid estimating jointly the market prices of risk related to the base, spike and drop regime from very limited data. On the other hand, in the obtained formulas for derivatives prices the 
parts related to different regimes are easily separated and, as suggested by De Jong and Huisman (2002), the spike/drop part can be multiplied by some factor to increase/ decrease the price of the spike/drop risk. Nevertheless, finding such a pricing measure that would also allow for a change in spikes and drops distribution is a challenging problem. A promising approach was used by Benth and Sgarra (2012), who applied the Escher transform theory in order to derive the market price of risk. Unfortunately, the Escher transform technique is applicable only for Levy processes, while the MRS model analyzed in this paper does not have independent increments and hence is not a Levy process.

We start the valuation of derivatives from finding the spot price dynamics under $\lambda$ parametrization. Let $\lambda(u)$ be a deterministic function square-integrable on $u \in$ $\left[0, T_{\max }\right]$, where $T_{\max }$ is a time horizon long enough to contain all maturities of derivatives quoted in the market, and introduce a new process $W_{t}^{\lambda}$ :

$$
W_{t}^{\lambda}=W_{t}+\int_{0}^{t} \frac{\lambda(u)}{\sigma_{b}} d u
$$

where $\sigma_{b}$ is the volatility of the base regime. From the Girsanov theorem (see Girsanov 1960) we have that $W_{t}^{\lambda}$ is a Wiener process under a new measure $Q^{\lambda}$ defined as a Radon-Nikodym derivative (Nikodym 1930)

$$
\frac{d Q^{\lambda}}{d Q}=\exp \left[-\int_{0}^{T_{\max }} \frac{\lambda(u)}{\sigma_{b}} d W_{u}-\frac{1}{2} \int_{0}^{T_{\max }}\left(\frac{\lambda(u)}{\sigma_{b}}\right)^{2} d u\right]
$$

with the filtration $\mathcal{F}_{t}^{W}$, being the natural filtration of the process $W_{t}$.

Now, the base regime process $X_{t, b}$ can be rewritten as:

$$
d X_{t, b}=\left[\alpha-\lambda(t)-\beta X_{t, b}\right] d t+\sigma_{b} d W_{t}^{\lambda}
$$

and the expected future spot price is given by:

$$
\begin{aligned}
E^{\lambda}\left(P_{t} \mid \mathcal{F}_{0}\right)= & p_{b b}^{(t)}\left[X_{0} e^{-\beta t}+\frac{\alpha}{\beta}\left(1-e^{-\beta t}\right)-\int_{0}^{t} e^{-\beta(t-u)} \lambda(u) d u\right] \\
& +p_{b s}^{(t)}\left(e^{\mu_{s}+\frac{1}{2} \sigma_{s}^{2}}+c_{s}\right)+p_{b d}^{(t)}\left(-e^{\mu_{d}+\frac{1}{2} \sigma_{d}^{2}}+c_{d}\right)+g_{t} .
\end{aligned}
$$

The function $\lambda(T)$ can be calibrated to the market forward prices so that $E^{\lambda}\left(P_{T} \mid \mathcal{F}_{0}\right)=f_{0}^{T}$, e.g. by using some fitting procedure (like the least squares minimization). Alternatively, one can find the risk premium and then use the relation between the market price of risk $\lambda(T)$ and the risk premium:

$$
p_{b b}^{(T)} \int_{0}^{T} e^{-\beta(T-u)} \lambda(u) d u=R P(T),
$$


which is a simple consequence of the fact that $R P(T)=E\left(P_{T} \mid \mathcal{F}_{0}\right)-E^{\lambda}\left(P_{T} \mid \mathcal{F}_{0}\right)$, formula (18) and Ito's lemma.

Now, the price of a European call option written on the electricity spot price can be derived.

Option price formula If the electricity spot price $P_{t}$ is given by the MRS model defined by Eqs. (1)-(7), then the price of a European call option written on $P_{t}$ with strike price $K$ and maturity $T$ is equal to:

$$
C_{T}(K)=e^{-r T}\left[p_{b b}^{(T)} C_{T, b}(K)+p_{b s}^{(T)} C_{T, s}(K)+p_{b d}^{(T)} C_{T, d}(K)\right]
$$

where

$$
\begin{aligned}
C_{T, b}(K)= & \frac{s}{\sqrt{2 \pi}} \exp \left(-\frac{\left(K^{\prime}-m\right)^{2}}{2 s^{2}}\right)+\left(m-K^{\prime}\right)\left[1-\Phi\left(\frac{K^{\prime}-m}{s}\right)\right], \\
C_{T, s}(K)= & \mathbb{I}_{\left\{K^{\prime}>c_{s}\right\}}\left\{\exp \left(\mu_{s}+\frac{\sigma_{s}^{2}}{2}\right)\left[1-\Phi\left(\frac{\log \left(K^{\prime}-c_{s}\right)-\mu_{s}-\sigma_{s}^{2}}{\sigma_{s}}\right)\right]\right. \\
& \left.-\left(K^{\prime}-c_{s}\right)\left[1-F_{L N\left(\mu_{s}, \sigma_{s}^{2}\right)}\left(K^{\prime}-c_{s}\right)\right]\right\} \\
& +\mathbb{I}_{\left\{K^{\prime} \leq c_{s}\right\}}\left[\exp \left(\mu_{s}+\frac{\sigma_{s}^{2}}{2}\right)+c_{s}-K^{\prime}\right]
\end{aligned}
$$

and

$$
\begin{aligned}
C_{T, d}(K)= & \mathbb{I}_{\left\{K^{\prime}<c_{d}\right\}}\left\{-\exp \left(\mu_{d}+\frac{\sigma_{d}^{2}}{2}\right) \Phi\left[\frac{\log \left(c_{d}-K^{\prime}\right)-\mu_{d}-\sigma_{d}^{2}}{\sigma_{d}}\right]\right. \\
& \left.+\left(c_{d}-K^{\prime}\right) F_{L N\left(\mu_{d}, \sigma_{d}^{2}\right)}\left(c_{d}-K^{\prime}\right)\right\} .
\end{aligned}
$$

Further, $K^{\prime}=K-g_{T}, m=X_{0} e^{-\beta T}+\frac{\alpha}{\beta}\left(1-e^{-\beta T}\right)-\int_{0}^{T} e^{-\beta(T-u)} \lambda(u) d u, s^{2}=$ $\frac{\sigma_{b}^{2}}{2 \beta}\left(1-e^{-2 \beta T}\right)$ and $F_{L N\left(\mu, \sigma^{2}\right)}$ is the cumulative distribution function of the lognormal distribution with parameters $\mu$ and $\sigma^{2}$. Note that, in order to make the exposition of the paper clear, the price derivation is moved to the "Appendix".

Here, we assume that the option is settled in an infinitesimal period of time $[T, T+\Delta]$. However, in practice, the electricity spot price usually corresponds to a delivery during some period of time (e.g. an hour, a day) and, hence, the maturity of the option should be specified on the same time-scale. On the other hand, the analyzed spot price quotations usually represent some delivery period. For instance, if the considered data is quoted daily, as it will be in the empirical example of Sect. 5, then the maturity of the option would be also given in daily time-scale and would correspond to daily delivery. 


\subsection{Electricity forward contracts}

Probably, the most popular electricity derivatives are the forward contracts. Recall that a forward contract is an agreement to buy (sell) a certain amount of the underlying (here MWh of electricity) at a specified future date. Settlement of the contract can be specified in two ways: with physical delivery of electricity or with only financial clearing. Both types of settlement are in the following called delivery. Denote the price at time $t$ of a forward contract with a delivery at time $T$ by $f_{t}^{T}$. Since the cost of entering a forward contract is equal to zero, the expected future payoff under the pricing measure should fulfill:

$$
E^{\lambda}\left(P_{T}-f_{t}^{T} \mid \mathcal{F}_{t}\right)=0
$$

what implies that

$$
f_{t}^{T}=E^{\lambda}\left(P_{T} \mid \mathcal{F}_{t}\right)
$$

Observe, that now we define the price of a forward contract at any future date $t$. This is motivated by the fact that the valuation at time 0 of an option written on a forward contract requires the knowledge about the forward price dynamics at the option's maturity $t$.

Forward price formula If the electricity spot price $P_{t}$ is given by the MRS model defined by Eqs. (1)-(7), then the price at time $t$ of a forward contract written on $P_{t}$ with a delivery at time $T$ is given by the following formula

$$
\begin{aligned}
f_{t}^{T}= & P\left(R_{\lfloor T\rfloor}=b \mid \mathcal{F}_{t}\right)\left[E^{\lambda}\left(X_{t, b} \mid \mathcal{F}_{t}\right) e^{-\beta(T-t)}+\frac{\alpha}{\beta}\left(1-e^{-\beta(T-t)}\right)\right. \\
& \left.-\int_{t}^{T} e^{-\beta(T-u)} \lambda(u) d u\right]+P\left(R_{\lfloor T\rfloor}=s \mid \mathcal{F}_{t}\right)\left(e^{\mu_{s}+\frac{1}{2} \sigma_{s}^{2}}+c_{s}\right) \\
& +P\left(R_{\lfloor T\rfloor}=d \mid \mathcal{F}_{t}\right)\left(c_{d}-e^{\mu_{d}+\frac{1}{2} \sigma_{d}^{2}}\right)+g_{T},
\end{aligned}
$$

where $P\left(R_{\lfloor T\rfloor}=i \mid \mathcal{F}_{t}\right)=\sum_{j \in\{b, s, d\}} P\left(R_{\lfloor T\rfloor}=i \mid R_{\lfloor t\rfloor}=j\right) \mathbb{I}_{\left\{R_{\lfloor t\rfloor}=j\right\}}$.

Note that in the above formula $E^{\lambda}\left(X_{t, b} \mid \mathcal{F}_{t}\right)$ is used, since this expectation depends on the state process value at time $t$. Namely, if $R_{t}=b$ then $E^{\lambda}\left(X_{t, b} \mid \mathcal{F}_{t}\right)=X_{t, b}=$ $X_{t}$. On the other hand, if at time $t$ a spike or a drop occurred then $E^{\lambda}\left(X_{t, b} \mid \mathcal{F}_{t}\right)=$ $E^{\lambda}\left(X_{t, b} \mid \mathcal{F}_{t-1}\right)$ and again this expectation is dependent on $R_{t-1}$ value. A general formula for $E^{\lambda}\left(X_{t, b} \mid \mathcal{F}_{t}\right)$ can be found using the same derivations as in Remark 1.

When deriving the forward price dynamics, we have to remember that the properties of the obtained model should comply with the observed market prices. One of the most pronounced features of the market forward prices is the observed term structure of volatility, called the Samuelson effect. Precisely, the volatility of the forward prices is quite low for distant delivery periods, however, it increases rapidly with approaching 
maturity of the contracts. Here, the forward price volatility is described by the part $P\left(R_{\lfloor T\rfloor}=b \mid \mathcal{F}_{t}\right) E^{\lambda}\left(X_{t, b} \mid \mathcal{F}_{t}\right) e^{-\beta(T-t)}$ of formula (26). Hence, it is specified by the volatility of the spot price base regime scaled with $e^{-\beta(T-t)}$ and the corresponding probability of switching to the base regime. Observe that the scaling factor $e^{-\beta(T-t)}$ exhibits the Samuelson effect as it increases to 1 with $t$ approaching maturity time $T$. Moreover the forward price volatility, again due to the scaling factor, is lower than the spot price volatility. This is in compliance with the behavior of the market spot and forward prices.

Electricity forward contracts listed on energy exchanges are usually settled during a certain period of time (a week, a month, a year, etc.). Denote the price at time $t$ of a forward contract settled during the period $\left[T_{1}, T_{2}\right]$ by $f_{t}^{\left[T_{1}, T_{2}\right]}$. Obviously, the latter is the mean price of forward contracts with delivery during the period $\left[T_{1}, T_{2}\right]$, namely:

$$
f_{t}^{\left[T_{1}, T_{2}\right]}=\int_{T_{1}}^{T_{2}} w\left(T_{1}, T_{2}, T\right) f_{t}^{T} d T=\int_{T_{1}}^{T_{2}} w\left(T_{1}, T_{2}, T\right) E^{\lambda}\left(P_{T} \mid \mathcal{F}_{t}\right) d T
$$

where $w\left(T_{1}, T_{2}, T\right)$ is the weight function representing the time value of money. The form of $w$ depends on the contract specification. For contracts settled at maturity we have $w\left(T_{1}, T_{2}, T\right)=\frac{1}{T_{2}-T_{1}}$, while for instant settlement $w\left(T_{1}, T_{2}, T\right)=\frac{r e^{-r T}}{e^{-r T_{1}-e^{-r T_{2}}}}$, where $r>0$ is the interest rate (Benth et al. 2008a). The price $f_{t}^{\left[T_{1}, T_{2}\right]}$ can be obtained from formulas (26) and (27). Indeed, we have:

$$
\begin{aligned}
f_{t}^{\left[T_{1}, T_{2}\right]}= & E^{\lambda}\left(X_{t, b} \mid \mathcal{F}_{t}\right) \int_{T_{1}}^{T_{2}} w\left(T_{1}, T_{2}, T\right) P\left(R_{\lfloor T\rfloor}=b \mid \mathcal{F}_{t}\right) e^{-\beta(T-t)} d T \\
& +\int_{T_{1}}^{T_{2}} w\left(T_{1}, T_{2}, T\right) P\left(R_{\lfloor T\rfloor}=b \mid \mathcal{F}_{t}\right) \\
& \times\left[\frac{\alpha}{\beta}\left(1-e^{-\beta(T-t)}\right)-\int_{t} e^{-\beta(T-u)} \lambda(u) d u\right] d T \\
& +\left(e^{\mu_{s}+\frac{1}{2} \sigma_{s}^{2}}+c_{s}\right) \int_{T_{1}}^{T_{2}} w\left(T_{1}, T_{2}, T\right) P\left(R_{\lfloor T\rfloor}=s \mid \mathcal{F}_{t}\right) d T \\
& +\left(c_{d}-e^{\mu_{d}+\frac{1}{2} \sigma_{d}^{2}}\right) \int_{T_{1}}^{T_{2}} w\left(T_{1}, T_{2}, T\right) P\left(R_{\lfloor T\rfloor}=d \mid \mathcal{F}_{t}\right) d T \\
& +\int_{T_{1}}^{T_{2}} w\left(T_{1}, T_{2}, T\right) g_{T} d T .
\end{aligned}
$$




\subsection{Options written on electricity forward contracts}

Finally, we find an explicit formula for a European call option written on a forward contract delivering electricity during a specified period of time. Observe, that the forward price $f_{t}^{\left[T_{1}, T_{2}\right]}$ depends on the spot price at time $t$ and, as a consequence, also on the state process value at time $t$.

We consider an option written on an electricity forward contract with settlement during a specified period of time, as it is the most popular specification of electricity options on energy exchanges. For example, in the EEX market there are options written on forward contracts with monthly, quarterly and yearly settlement periods. The maturity of such options is set to the fourth business day before the beginning of the underlying contracts settlement period. The EEX example will be examined in Sect. 5.

Price formula for an option written on a forward contract If the electricity spot price $P_{t}$ is given by the model defined by Eqs. (1)-(7), then the price of a European call option with strike price $K$ and maturity $t$ written on a forward contract with delivery during the period $\left[T_{1}, T_{2}\right]$ is equal to:

$$
\begin{aligned}
C f_{t}^{\left[T_{1}, T_{2}\right]}(K)= & e^{-r t}\left\{A_{0}(b) C_{t, b}\left(\frac{K-B_{0}(b)}{A_{0}(b)}+g_{t}\right) P\left(R_{\lfloor t\rfloor}=b \mid R_{0}=b\right)\right. \\
& +\sum_{i \in\{s, d\}} \sum_{k=1}^{\lfloor t\rfloor}\left[A_{k}(i) C_{\lfloor t\rfloor-k+1, b}\left(\frac{K-B_{k}(i)}{A_{k}(i)}+g_{\lfloor t\rfloor-k+1}\right)\right. \\
& \left.\left.\times P\left(R_{\lfloor t\rfloor}=i, R_{\lfloor t\rfloor-1} \neq b, \ldots, R_{\lfloor t\rfloor-k}=b \mid R_{0}=b\right)\right]\right\},
\end{aligned}
$$

where

$$
\begin{aligned}
A_{k}(i)= & \int_{T_{1}}^{T_{2}} w\left(T_{1}, T_{2}, T\right) P\left(R_{\lfloor T\rfloor}=b \mid R_{\lfloor t\rfloor}=i\right) e^{-\beta(T-\lfloor t\rfloor+k-1)} d T \\
A_{0}(b)= & \int_{T_{1}}^{T_{2}} w\left(T_{1}, T_{2}, T\right) P\left(R_{\lfloor T\rfloor}=b \mid R_{\lfloor t\rfloor}=b\right) e^{-\beta(T-t)} d T \\
B_{k}(i)= & \int_{T_{1}}^{T_{2}} w\left(T_{1}, T_{2}, T\right) P\left(R_{\lfloor T\rfloor}=b \mid R_{\lfloor t\rfloor}=i\right) \\
& \times\left[\frac{\alpha}{\beta}\left(1-e^{-\beta(T-\lfloor t\rfloor+k-1)}\right)-\int_{\lfloor t\rfloor-k+1}^{T} e^{-\beta(T-u)} \lambda(u) d u\right] d T \\
& +\left(e^{\mu_{s}+\frac{1}{2} \sigma_{s}^{2}}+c_{s}\right) \int_{T_{1}}^{T_{2}} w\left(T_{1}, T_{2}, T\right) P\left(R_{\lfloor T\rfloor}=s \mid R_{\lfloor t\rfloor}=i\right) d T
\end{aligned}
$$




$$
\begin{aligned}
& +\left(c_{d}-e^{\mu_{d}+\frac{1}{2} \sigma_{d}^{2}}\right) \int_{T_{1}}^{T_{2}} w\left(T_{1}, T_{2}, T\right) P\left(R_{\lfloor T\rfloor}=d \mid R_{\lfloor t\rfloor}=i\right) d T \\
& +\int_{T_{1}}^{T_{2}} w\left(T_{1}, T_{2}, T\right) g_{T} d T, \\
B_{0}(b)= & \int_{T_{1}}^{T_{2}} w\left(T_{1}, T_{2}, T\right) P\left(R_{\lfloor T\rfloor}=b \mid R_{\lfloor t\rfloor}=b\right) \\
& \times\left[\frac{\alpha}{\beta}\left(1-e^{-\beta(T-t)}\right)-\int_{t} e^{-\beta(T-u)} \lambda(u) d u\right] d T \\
& +\left(e^{\mu_{s}+\frac{1}{2} \sigma_{s}^{2}}+c_{s}\right) \int_{T_{1}}^{T_{2}} w\left(T_{1}, T_{2}, T\right) P\left(R_{\lfloor T\rfloor}=s \mid R_{\lfloor t\rfloor}=b\right) d T \\
& +\left(c_{d}-e^{\left.\mu_{d}+\frac{1}{2} \sigma_{d}^{2}\right)} \int_{T_{1}}^{T_{2}} w\left(T_{1}, T_{2}, T\right) P\left(R_{\lfloor T\rfloor}=d \mid R_{\lfloor t\rfloor}=b\right) d T\right. \\
& \int_{T_{1}}^{T_{2}} w\left(T_{1}, T_{2}, T\right) g_{T} d T .
\end{aligned}
$$

and $C_{t, b}(K)$ is the 'base regime part' of the price of a European call option written on the electricity spot price with maturity $t$ and strike $K$, see Eq. (21) with $T=t$.

\section{EEX market example}

Theoretical results from the previous sections allow us to price energy derivatives. We assume that the electricity spot price follows the model specified by Eqs. (1)-(7) with a periodic transition matrix and $c_{s}, c_{d}$ being the first and third quartile of the stochastic part [i.e. $X_{t}$ in Eq. (1)] of the dataset, respectively. We use mean daily EEX spot prices from the period January 2, 2006-January 2, 2011 (5 years and 261 whole weeks). In order to calibrate the model, we first remove the seasonal component.

We assume that the deterministic function $g_{t}$ is composed of two parts: a long term trend $L_{t}$ and a weekly seasonality $S_{t}$. Since the valuation of derivatives requires forecasting the seasonal component, we use the standard approach (see e.g. Bierbrauer and Menn 2007; Geman and Roncoroni 2006; Weron 2008) and model the long term trend by a sum of sine functions:

$$
\begin{aligned}
L_{t}= & \left(a_{1}+a_{2} t\right) \sin \left[2 \pi\left(t+a_{3}\right)\right] \\
& +\left(a_{4}+a_{5} t\right) \sin \left[2 \pi a_{6}\left(t+a_{7}\right)\right]+a_{8}+a_{9} t+a_{10} t^{2},
\end{aligned}
$$


Table 1 Coefficients of the function $L_{t}$, see Eq. (34), fitted to the EEX spot prices

\begin{tabular}{cccccccccc}
\hline$a_{1}$ & $a_{2}$ & $a_{3}$ & $a_{4}$ & $a_{5}$ & $a_{6}$ & $a_{7}$ & $a_{8}$ & $a_{9}$ & $a_{10}$ \\
\hline-11.99 & 0.55 & -0.13 & 34.03 & -8.04 & 0.46 & 6.75 & 25.37 & 19.20 & -3.35 \\
\hline
\end{tabular}
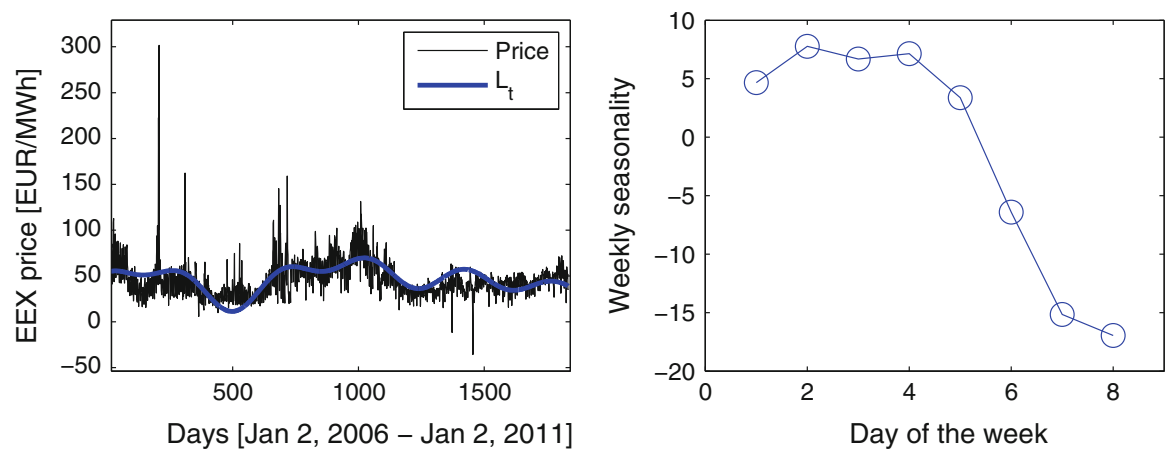

Fig. 1 Left panel EEX spot prices and the fitted long term seasonal component (blue solid line). Right panel weekly periodicity calculated for the EEX spot prices. Note, that $1-$ Monday, 2-Tuesday, ..., 7-Sunday, while the 8 day of the week is used for the German national holidays. (Color figure online)

where $t$ is in yearly time scale. Note, that the first component of the above sum is responsible for the yearly periodicity, while the second one captures seasonalities of different period than one year (here, we obtain nearly half-year period, see $a_{6}$ in Table 1). The function $L_{t}$ is fitted to the EEX spot prices using the least squares method. The obtained curve is plotted in Fig. 1, while the obtained $a_{i}$ coefficients are given in Table 1.

The estimated long term trend is subtracted from the analyzed time series. Next, the short term seasonal component is estimated using the 'average week' method, being equivalent to using dummy variables (see e.g. Cartea and Figueroa 2005; De Jong 2006). Namely, we calculate the mean of prices corresponding to each day of the week (German national holidays are treated as the eight day of the week). The obtained weekly pattern is plotted in Fig. 1.

Finally, the deseasonalized prices are obtained by subtracting the long and short term trend from the EEX spot prices. Moreover, the data is shifted so that the minimum of the deseasonalized and the original prices is the same. The resulting time series can be seen in Fig. 2.

After removing seasonality, we are left with modeling the stochastic part $X_{t}$. Here, we calibrate the 3-regime MRS model, see Eqs. (2)-(7), to the deseasonalized EEX prices. To this end, we use a version of the Expectation-Maximization algorithm of Dempster et al. (1977), which was applied to MRS models by Hamilton (1990) and was later refined by Kim (1994). It is a two-step iterative procedure:

- Step 1: For a parameter vector $\theta$ compute the conditional probabilities for the process being in regime $j$ at time $t, P\left(R_{t}=j \mid X_{1}, X_{2}, \ldots, X_{N} ; \theta\right)$, where $\left(X_{1}, X_{2}, \ldots, X_{N}\right)$ is a sample of observations. 


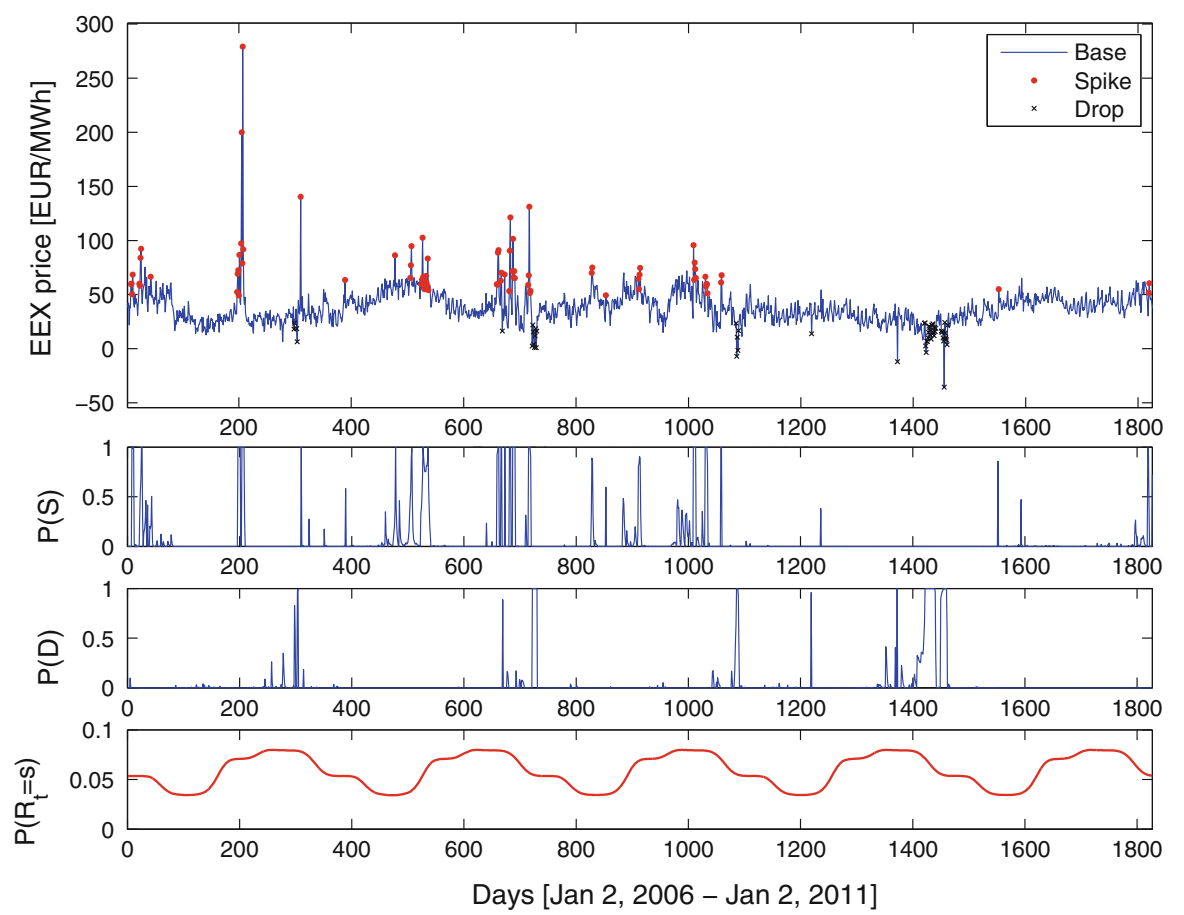

Fig. 2 Calibration results of the 3-regime MRS model fitted to the EEX deseasonalized prices. The prices classified to the spike regime i.e. with $P(S)=P\left(R_{t}=s \mid X_{1}, X_{2}, \ldots, X_{N}\right)>0.5$ are denoted by red dots, while the prices classified to the drop regime i.e. with $P(D)=P\left(R_{t}=d \mid X_{1}, X_{2}, \ldots, X_{N}\right)>0.5$ are denoted with black $X$ 's. Additionally, in the lower panels the corresponding probabilities are plotted. The estimated unconditional probability of spike occurrence is displayed in the bottom panel. Observe, that the highest probability of spike is obtained for the Autumn/Winter period, while the lowest for Spring. (Color figure online)

- Step 2: Calculate new and more exact maximum likelihood estimates of $\theta$ using the likelihood function, weighted with the probabilities from step 1.

The steps 1 and 2 are repeated until a local maximum of the likelihood function is found. For the detailed description of the algorithm in case of the 3-regime model considered in this paper see the recent work of Janczura and Weron (2012). The obtained parameters are given in Table 2. Observe high probabilities of staying in the same regime, ranging from 0.40 for the drop regime up to 0.97 for the base regime. Hence, the assumed model allows for modeling consecutive spikes or drops in a very natural way. The calibration results are plotted in Fig. 2, where additionally the regimes classification is illustrated. As we may observe, spikes/drops occur usually as a series of high/low prices rather than separate outstanding observations. What is interesting to note, is the clear seasonal pattern in the estimated probability of spike, see the bottom panel in Fig. 2. Indeed, the highest spike occurrence probability is obtained for the Autumn/Winter season, while the lowest for Spring.

In order to validate the used MRS model, we apply a Kolmogorov-Smirnov goodness-of-fit test for the marginal distribution of the individual regimes, as well as, for the whole model. We use two testing procedures. The first one (called 
Table 2 Parameter estimates of the 3-regime MRS model fitted to the deseasonalized EEX prices

\begin{tabular}{|c|c|c|c|c|c|c|c|c|c|}
\hline \multicolumn{7}{|c|}{ Parameters } & \multicolumn{3}{|c|}{ Probabilities } \\
\hline$\alpha_{1}$ & $\beta_{1}$ & $\sigma_{1}^{2}$ & $\alpha_{2}$ & $\sigma_{2}^{2}$ & $\alpha_{3}$ & $\sigma_{3}^{2}$ & $p_{11}$ & $p_{22}$ & $p_{33}$ \\
\hline 5.98 & 0.16 & 39.53 & 2.89 & 0.64 & 2.62 & 0.33 & 0.97 & 0.66 & 0.40 \\
\hline
\end{tabular}

Table $3 p$ values of the goodness-of-fit tests for the 3-regime MRS model fitted to the deseasonalized EEX prices

\begin{tabular}{|c|c|c|c|c|c|c|c|c|}
\hline \multirow[b]{2}{*}{ Regime } & \multicolumn{4}{|c|}{ ewedf } & \multicolumn{4}{|l|}{ wedf } \\
\hline & Base & Spike & Drop & Model & Base & Spike & Drop & Model \\
\hline$p$ value & 0.64 & 0.16 & 0.50 & 0.34 & 0.30 & 0.59 & 0.95 & 0.25 \\
\hline
\end{tabular}

The test results for the ewedf, as well as, the wedf approach are provided

Table 4 Specification of the monthly forward contracts listed on the EEX market on January 3,2011

\begin{tabular}{llll}
\hline Name & Settlement price & $T_{1}$ & $T_{2}$ \\
\hline Feb-11 & 54.35 & 1.2 .2011 & 28.2 .2011 \\
Mar-11 & 51.64 & 1.3 .2011 & 31.3 .2011 \\
Apr-11 & 48.07 & 1.4 .2011 & 30.4 .2011 \\
May-11 & 45.53 & 1.5 .2011 & 31.5 .2011 \\
Jun-11 & 48.50 & 1.6 .2011 & 30.6 .2011 \\
Jul-11 & 49.00 & 1.7 .2011 & 31.7 .2011 \\
\hline
\end{tabular}

ewedf-equally weighted empirical distribution function) is based on classifying observations to the most probable regime, i.e. assuming that $R_{t}=i$ if $P\left(R_{t}=\right.$ $\left.i \mid X_{1}, X_{2}, \ldots, X_{N}\right)>0.5$. Note that, due to the cutoff values $c_{s}$ and $c_{d}$, if a probability of spike (drop) is positive, the probability of drop (spike) must be equal to 0 . Hence, an observation is considered a spike or a drop if it is the most probable regime for it. As a consequence, the standard Kolmogorov-Smirnov goodness-of-fit test can be applied. The second one (called wedf) utilizes a notion of the weighted empirical distribution function, where $t$-th observation is taken into account with weight proportional to the probability $P\left(R_{t}=i \mid X_{1}, X_{2}, \ldots, X_{N}\right)$. For the detailed testing procedure derivation see Janczura and Weron (2013). The obtained test $p$ values are given in Table 3. Recall, that $p$ value higher than $5 \%$ means that we cannot reject, at the $5 \%$ significance level, the hypothesis that the analyzed dataset was driven by the assumed model. As all of the obtained $p$ values are higher than $5 \%$, we cannot reject the considered 3-regime MRS model as a proper one for the analyzed dataset.

Before we start with the valuation of derivatives we have to find the risk premium and the function $\lambda$, see Eq. (19). We use monthly forward contracts listed on the EEX market on January 3, 2011, i.e. on the day directly following the calibration period. The prices, as well as, the delivery periods of the analyzed forward contracts are given in Table 4. Since we analyze monthly contracts, the risk premium should be also calculated on the monthly basis, i.e. instead of Eq. (8) we use: 


$$
R P\left(T_{1}, T_{2}\right)=\frac{1}{T_{2}-T_{1}+1} \sum_{t=T_{1}}^{T_{2}} E\left(P_{t} \mid \mathcal{F}_{0}\right)-f_{0}^{\left[T_{1}, T_{2}\right]}
$$

with $f_{0}^{\left[T_{1}, T_{2}\right]}$ being the monthly forward contracts quotations (see Table 4) and $E\left(P_{t} \mid \mathcal{F}_{0}\right)$ being the forecast of the future spot price based on the fitted model. Note that the average monthly expected spot price is calculated as an arithmetic mean instead of an integral, because the analyzed spot prices are quoted is discrete time (on a daily basis). The values of the risk premium obtained from contracts with different delivery periods are plotted in Fig. 3 (for contract specifications see Table 4).

The negative risk premium [if defined as in (8)] means that the forward prices are higher than the expected spot prices, what might indicate that there is high demand for the forward contracts. Such situation occurs if buyers (retailers) are risk averse and are willing to pay more to ensure a future delivery. On the other hand, according to the normal backwardation theory, a positive risk premium means that producers accept forward prices lower than the expected spot prices in order to secure consumption of electricity produced in the future. It should be noted that there are also different attempts to explain signs of the risk premium in the literature. Benth and MeyerBrandis (2009) use an information-based approach, while Benth et al. (2008b) give an interpretation of the risk premium on the basis of the certainty equivalent principle and jumps in the spot price dynamics.

Here, we observe an evidence for the negative risk premium, especially for contracts with approaching delivery period, see Fig. 3. For the contracts with more distant delivery period the obtained risk premium is less significant. Since calibrating the risk premium on only one day might not be representative, we also calculate the risk premium for the same contracts but on the next three Mondays of January 2011, namely on 10th, 17th and 24th of January. The obtained values are plotted in Fig. 7 in the "Appendix". Observe, that although the values change for different times of calculation, what might be due to the low liquidity of the contracts, the shape of the risk premium is generally preserved. For each case the risk premium is negative and increase with increasing time to delivery. The obtained term structure of risk premium might indicate that for short deliveries the risk-averse retailers dominate on the market

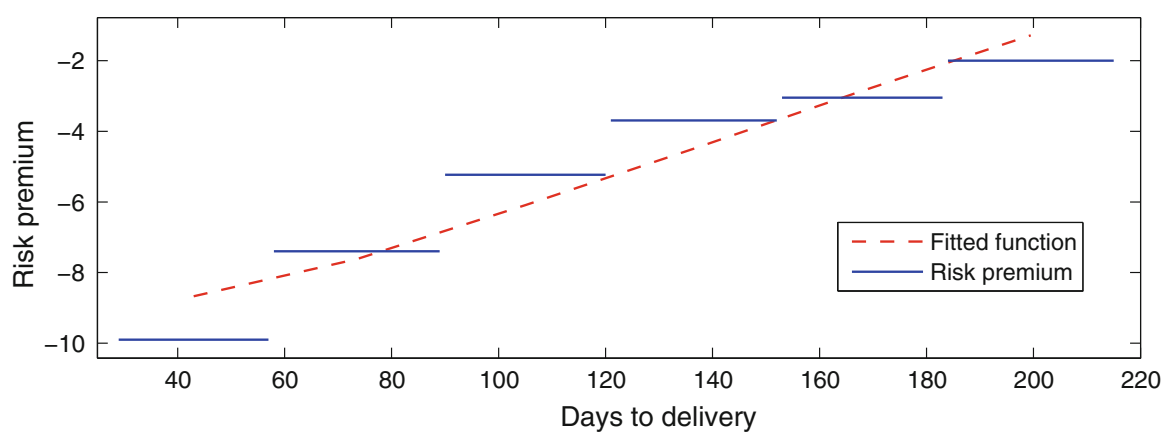

Fig. 3 The risk premium obtained from monthly forward contracts with different delivery periods (blue lines), as well as the fitted function in the case of linear $\lambda(t)$ [see Eq. (36)] plotted with red dashed line. (Color figure online) 


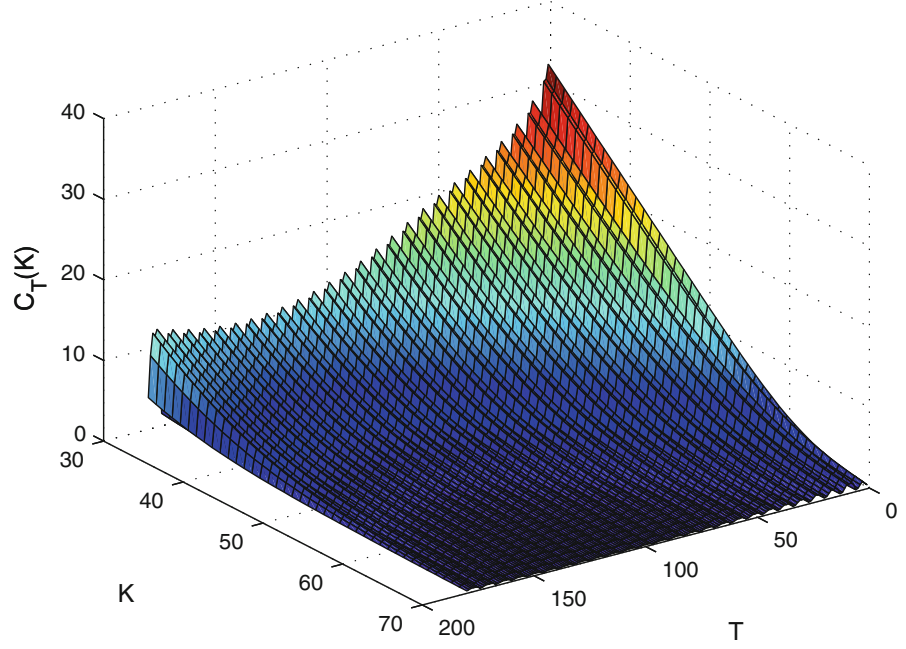

Fig. 4 Prices of a European call option written on the electricity spot price for different maturities $T$ and strike prices $K$. The prices are calculated on January 3, 2011

hedging against a possible spike risks, while with increasing time of delivery the forward contracts become more attractive for electricity producers. Similar results were also obtained by other authors, like e.g. Geman and Vasciek (2001) for the case of the PJM market or Ronn and Wimschulte (2009) for the EEX market (note that these authors define the risk premium as the difference between the forward prices and the expected spot prices, which is $-R P(T)$ defined in this article).

Next, using the obtained risk premium values we fit the function $\lambda$, i.e the market price of risk, see Eq. (19). To this end, we assume that the market price of risk $\lambda(t)=$ $\lambda_{1} t+\lambda_{2}$. Again, since we analyze monthly forward contracts and the risk premium is found on the monthly basis, $\lambda_{1}$ and $\lambda_{2}$ are found by fitting:

$$
\frac{\lambda_{1} \sum_{t=T_{1}}^{T_{2}} p_{b b}^{(t)}\left(t-\left(1-e^{-\beta t}\right) / \beta\right)+\lambda_{2} \sum_{t=T_{1}}^{T_{2}} p_{b b}^{(t)}\left(1-e^{-\beta t}\right)}{\beta\left(T_{2}-T_{1}+1\right)}=R P\left(T_{1}, T_{2}\right)
$$

See Eq. (19) for the comparison with the continuous time scale. Using the leastsquares minimization scheme we get $\lambda(t)=0.0084 t-1.8387$, see the red dashed line in Fig. 3 for the plot of the function fitted to the risk premium [i.e. the left hand side of Eq. (36)]. Note that, the choice of the linear form of the $\lambda$ function allows to model dependence between the risk premium and time to maturity. Obviously, alternative functions can be used as well. Different time dependent market price of risk specifications were studied e.g. by Haerdle and Lopez-Cabrera (2012) in the case of weather derivatives. It was found that the market price of risk shows seasonal variations and is an increasing function of the futures expiration date. However, for the example studied in the paper, the linear function yields a reasonable fit as compared to a constant, see Figs. 3 and 6, at the same time being simple enough to be numerically and analytically easily handled. 


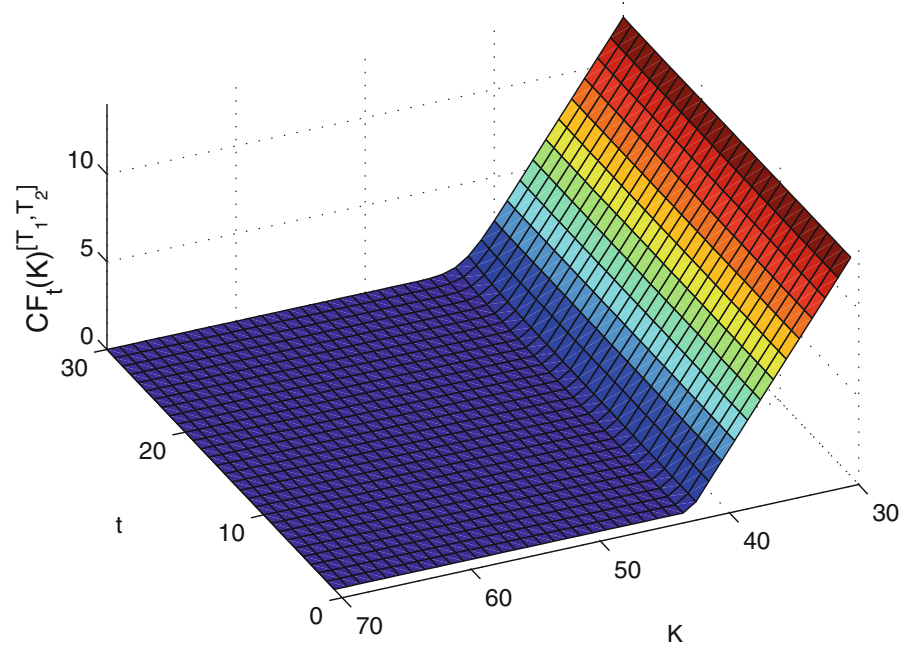

Fig. 5 Prices of a European call option written on the electricity forward contract with settlement in February 2011 for different maturities $t$ and strike prices $K$. The prices are calculated on January 3, 2011

Table 5 Prices of European call options written on monthly forward contracts with strike price $K=30$

The prices are calculated on January 3, 2011

\begin{tabular}{llc}
\hline $\begin{array}{l}\text { Month of delivery } \\
{\left[T_{1}, T_{2}\right]}\end{array}$ & Options maturity $t$ & $\begin{array}{l}\text { Options price } \\
C f_{t}^{\left[T_{1}, T_{2}\right]}(K)\end{array}$ \\
\hline February & 26.1 .2011 & 13.5449 \\
March & 23.2 .2011 & 9.1074 \\
April & 28.3 .2011 & 4.2258 \\
May & 26.4 .2011 & 2.6826 \\
June & 28.5 .2011 & 3.6148 \\
July & 27.6 .2011 & 7.1913 \\
\hline
\end{tabular}

Now, we can derive the price of a European call option written on the electricity spot price. Assume that the interest rate $r$ is equal to 0 . The option prices obtained in Sect. 4.1 with different maturities $t$ and strike prices $K$ are plotted in Fig. 4. Obviously, the lower is the strike price, the higher is the call option price. What is interesting to note, is how the option price depends on the maturity tenor $T$. Observe the clear seasonal pattern of option prices, both on the weekly and the long-term level. The long-term seasonality is caused not only by the deterministic component $g_{t}$ but also by the periodic transition matrix allowing for varying spike (drop) probabilities during the year. Recall that in the EEX market the spike probability is the highest in Autumn/Winter and the lowest in Spring, see Fig. 2.

Now, we derive the prices of European call options written on monthly forward contracts. Using the results obtained in Sect. 4.3 we calculate the price of an option written on the forward contract with settlement in February 2011. The results are plotted in Fig. 5. In order to check how the option price varies according to the delivery period, we calculate the prices of options written on monthly forward contracts with 
deliveries within the next 6 months (i.e. February 2011 till July 2011). According to the products specification in the EEX market, the maturity of the options is set to the fourth business day before the beginning of the delivery period. The obtained option prices are given in Table 5. Similarly, as in the case of options written on the spot price, we observe the lowest option prices for settlement during the Spring months.

\section{Conclusions}

In this paper we have derived premiums of European options written on electricity spot, as well as, forward prices. We assumed that electricity spot prices can be described by a 3-regime MRS model with independent spikes and drops and periodic transition matrix, proposed earlier by Janczura and Weron (2010). The forward prices were then derived using the risk premium approach and fitting the model-based prices to the observed forward curve. Next, using the spot and forward price dynamics we calculated prices of the corresponding European options. It should be stressed that the presented formulas are valid only under assumption of the 3-regime MRS model of Janczura and Weron (2010). However, the presented methodology can be easily extended to other specifications of the MRS models.

The assumed model was then calibrated to the spot prices from the European Energy Exchange. We have validated the model choice by performing a statistical goodnessof-fit test. Next, using monthly forward contracts quotations we have calculated the risk premium. We have obtained negative values, especially significant for contracts with approaching maturity. For contracts with distant settlement the risk premium values were higher.

Finally, the presented methodology and the calibration results allowed us to find prices of European options currently listed on the EEX market. As the assumed 3-regime MRS model seems to be adequate to describe dynamics of electricity spot prices, the results of the paper can be used for pricing of electricity derivatives and, hence, yield an effective risk management tool.

Acknowledgments We thank Tomasz Piesiewicz from Tauron PE for the EEX spot price data. This work was supported by funds from the National Science Centre (NCN) through Grant No. 2011/01/B/HS4/01077.

Open Access This article is distributed under the terms of the Creative Commons Attribution License which permits any use, distribution, and reproduction in any medium, provided the original author(s) and the source are credited.

\section{Appendix}

Derivation of the option price formula Using standard arguments the option price is the discounted expected value of the payoff function under the pricing measure (see e.g. Musiela and Rutkowski (1997)). Moreover, analogously to formula (11) this expectation is equal to:

$$
C_{T}(K)=e^{-r T} E^{\lambda}\left[\left(P_{T}-K\right)^{+} \mid \mathcal{F}_{0}\right]=e^{-r T} E^{\lambda}\left[\left(X_{T}+g_{T}-K\right)^{+} \mid \mathcal{F}_{0}\right]
$$




$$
\begin{aligned}
= & e^{-r T}\left\{p_{b b}^{(T)} E^{\lambda}\left[\left(X_{T, b}-K^{\prime}\right)^{+} \mid \mathcal{F}_{0}\right]+p_{b s}^{(T)} E\left[\left(X_{\lfloor T\rfloor, s}-K^{\prime}\right)^{+} \mid \mathcal{F}_{0}\right]\right. \\
& \left.+p_{b d}^{(T)} E\left[\left(X_{\lfloor T\rfloor, d}-K^{\prime}\right)^{+} \mid \mathcal{F}_{0}\right]\right\},
\end{aligned}
$$

where $K^{\prime}=K-g_{T}$ and $(x)^{+}=\max (0, x)$.

We start with pricing the base regime part $C_{T, b}(K)$ :

$$
C_{T, b}(K)=E^{\lambda}\left[\left(X_{T, b}-K^{\prime}\right)^{+} \mid \mathcal{F}_{0}\right]=\int_{K^{\prime}}^{\infty}\left(x-K^{\prime}\right) f_{X_{T, b} \mid X_{0, b}}(x) d x,
$$

where $f_{X_{T, b} \mid X_{0, b}}(x)$ denotes the density of $X_{T, b}$ conditional on $X_{0, b}$. From (17) and Ito's lemma we have that:

$$
X_{T, b}=X_{0, b} e^{-\beta T}+\frac{\alpha}{\beta}\left(1-e^{-\beta T}\right)-\int_{0}^{T} e^{-\beta(T-u)} \lambda(u) d u+\sigma_{b} \int_{0}^{T} e^{-\beta(T-u)} d W_{u}^{\lambda} .
$$

Hence, $X_{T, b}$ given $X_{0, b}$ has a Gaussian distribution with mean

$$
E\left(X_{T, b} \mid \mathcal{F}_{0}\right)=X_{0, b} e^{-\beta T}+\frac{\alpha}{\beta}\left(1-e^{-\beta T}\right)-\int_{0}^{T} e^{-\beta(T-u)} \lambda(u) d u
$$

and variance

$$
\operatorname{Var}\left(X_{T, b} \mid \mathcal{F}_{0}\right)=\frac{\sigma_{b}^{2}}{2 \beta}\left(1-e^{-2 \beta T}\right)
$$

Denote the mean by $m$ and the variance by $s^{2}$. We have:

$$
\begin{aligned}
C_{T, b}(K) & =\frac{1}{\sqrt{2 \pi} s} \int_{K^{\prime}}^{\infty}\left(x-K^{\prime}\right) \exp \left(-\frac{(x-m)^{2}}{2 s^{2}}\right) d x \\
& =\frac{s}{\sqrt{2 \pi}} \exp \left(-\frac{\left(K^{\prime}-m\right)^{2}}{2 s^{2}}\right)+\left(m-K^{\prime}\right)\left[1-\Phi\left(\frac{K^{\prime}-m}{s}\right)\right] .
\end{aligned}
$$

Now, we turn to the pricing of the spike regime part. Observe that if $K^{\prime} \leq c_{s}$, then $E\left[\left(X_{\lfloor T\rfloor, s}-K^{\prime}\right)^{+} \mid \mathcal{F}_{0}\right]=E\left(X_{\lfloor T\rfloor, s}\right)-K^{\prime}$. Assume that $K^{\prime}>c_{s}$. Denote the 
log-normal pdf by $f_{L N\left(\mu_{s}, \sigma_{s}^{2}\right)}$ and the cdf by $F_{L N\left(\mu_{s}, \sigma_{s}^{2}\right)}$. Then

$$
\begin{aligned}
E\left[\left(X_{\lfloor T\rfloor, s}-K^{\prime}\right)^{+} \mid \mathcal{F}_{0}\right]= & \int_{K^{\prime}}^{\infty}\left(x-K^{\prime}\right) f_{L N\left(\mu_{s}, \sigma_{s}^{2}\right)}\left(x-c_{s}\right) d x \\
= & \exp \left(\mu_{s}+\frac{\sigma_{s}^{2}}{2}\right)\left\{1-\Phi\left[\frac{\log \left(K^{\prime}-c_{s}\right)-\mu_{s}-\sigma_{s}^{2}}{\sigma_{s}}\right]\right\} \\
& -\left(K^{\prime}-c_{s}\right)\left[1-F_{L N\left(\mu_{s}, \sigma_{s}^{2}\right)}\left(K^{\prime}-c_{s}\right)\right]
\end{aligned}
$$

and we have that

$$
\begin{aligned}
E\left[\left(X_{\lfloor T\rfloor, s}-K^{\prime}\right)^{+} \mid \mathcal{F}_{0}\right]= & \mathbb{I}_{\left\{K^{\prime}>c_{s}\right\}}\left\{\exp \left(\mu_{s}+\frac{\sigma_{s}^{2}}{2}\right)\right. \\
& \times\left[1-\Phi\left(\frac{\log \left(K^{\prime}-c_{s}\right)-\mu_{s}-\sigma_{s}^{2}}{\sigma_{s}}\right)\right] \\
& \left.-\left(K^{\prime}-c_{s}\right)\left[1-F_{L N\left(\mu_{s}, \sigma_{s}^{2}\right)}\left(K^{\prime}-c_{s}\right)\right]\right\} \\
& +\mathbb{I}_{\left\{K^{\prime} \leq c_{s}\right\}}\left[\exp \left(\mu_{s}+\frac{\sigma_{s}^{2}}{2}\right)+c_{s}-K^{\prime}\right] .
\end{aligned}
$$

Similarly, we can price the drop regime part:

$$
\begin{aligned}
E\left[\left(X_{\lfloor T\rfloor, d}-K^{\prime}\right)^{+} \mid \mathcal{F}_{0}\right]= & \mathbb{I}_{\left\{K^{\prime}<c_{d}\right\}}\left\{-\exp \left(\mu_{d}+\frac{\sigma_{d}^{2}}{2}\right)\right. \\
& \times \Phi\left[\frac{\log \left(c_{d}-K^{\prime}\right)-\mu_{d}-\sigma_{d}^{2}}{\sigma_{d}}\right] \\
& \left.+\left(c_{d}-K^{\prime}\right) F_{L N\left(\mu_{d}, \sigma_{d}^{2}\right)}\left(c_{d}-K^{\prime}\right)\right\} .
\end{aligned}
$$

Finally, letting $C_{T, i}(K)=E\left[\left(X_{\lfloor T\rfloor, i}-K^{\prime}\right)^{+} \mid \mathcal{F}_{0}\right]$ for $i \in\{s, d\}$ and combining formulas (37), (42), (44) and (45) yields the result.

Derivation of the forward price First, note that

$$
\begin{aligned}
E^{\lambda}\left(P_{T} \mid \mathcal{F}_{t}\right)= & E^{\lambda}\left(X_{T} \mid \mathcal{F}_{t}\right)+g_{T}=E^{\lambda}\left(\mathbb{I}_{\left\{R_{\lfloor T\rfloor}=b\right\}} X_{T, b}+\mathbb{I}_{\left\{R_{\lfloor T\rfloor}=s\right\}} X_{\lfloor T\rfloor, s}\right. \\
& \left.+\mathbb{I}_{\left\{R_{\lfloor T\rfloor}=d\right\}} X_{\lfloor T\rfloor, d} \mid \mathcal{F}_{t}\right)+g_{T} .
\end{aligned}
$$

Since $X_{\lfloor T\rfloor, i}$, for $i=s, d$, is independent of $R_{\lfloor T\rfloor}$ and $\mathcal{F}_{t}$, we have: $E^{\lambda}\left(\mathbb{I}_{\left\{R_{\lfloor T\rfloor}=i\right\}} X_{T, i} \mid\right.$ $\left.\mathcal{F}_{t}\right)=P\left(R_{\lfloor T\rfloor}=i \mid \mathcal{F}_{t}\right) E\left(X_{\lfloor T\rfloor, i}\right), i=s, d$. Secondly, we have

$$
\begin{aligned}
E^{\lambda}\left(\mathbb{I}_{\left\{R_{\lfloor T\rfloor}=b\right\}} X_{T, b} \mid \mathcal{F}_{t}\right)= & E^{\lambda}\left(\mathbb{I}_{\left\{R_{\lfloor T\rfloor}=b\right\}} \mid \mathcal{F}_{t}\right) E^{\lambda}\left(X_{T, b} \mid \mathcal{F}_{t}\right) \\
& +P\left(R_{\lfloor T\rfloor}=b \mid \mathcal{F}_{t}\right) E^{\lambda}\left(X_{T, b} \mid \mathcal{F}_{t}\right)
\end{aligned}
$$


and from the base regime definition and Ito's lemma:

$$
\begin{aligned}
E^{\lambda}\left(X_{T, b} \mid \mathcal{F}_{t}\right)= & E^{\lambda}\left(X_{t, b} \mid \mathcal{F}_{t}\right) e^{-\beta(T-t)}+\frac{\alpha}{\beta}\left(1-e^{-\beta(T-t)}\right) \\
& -\int_{t}^{T} e^{-\beta(T-u)} \lambda(u) d u .
\end{aligned}
$$

Moreover, from the definitions of the spike and drop regimes, see Eqs. (6) and (7), we get:

$$
E\left(X_{\lfloor T\rfloor, s}\right)=e^{\mu_{s}+\frac{1}{2} \sigma_{s}^{2}}+c_{s}
$$

and

$$
E\left(X_{\lfloor T\rfloor, d}\right)=c_{d}-e^{\mu_{d}+\frac{1}{2} \sigma_{d}^{2}}
$$

Finally, combining formula (46) with (48), (49) and (50) yields the result.

Derivation of the price formula for an option written on a forward contract We start the derivation of the option price formula with the following observation.

If $R_{\lfloor t\rfloor}=b$, then the forward price is given by:

$$
\begin{aligned}
f_{t \mid\left\{R_{\lfloor t\rfloor}\left[T_{1}, T_{2}\right]\right.}= & X_{t, b} \int_{T_{1}}^{T_{2}} w\left(T_{1}, T_{2}, T\right) P\left(R_{\lfloor T\rfloor}=b \mid R_{\lfloor t\rfloor}=b\right) e^{-\beta(T-t)} d T \\
& +\int_{T_{1}}^{T_{2}} w\left(T_{1}, T_{2}, T\right) P\left(R_{\lfloor T\rfloor}=b \mid R_{\lfloor t\rfloor}=b\right) \\
& \times\left[\frac{\alpha}{\beta}\left(1-e^{-\beta(T-t)}\right)-\int_{t} e^{-\beta(T-u)} \lambda(u) d u\right] d T \\
& +\left(e^{\mu_{s}+\frac{1}{2} \sigma_{s}^{2}}+c_{s}\right) \int_{T_{1}}^{T_{2}} w\left(T_{1}, T_{2}, T\right) P\left(R_{\lfloor T\rfloor}=s \mid R_{\lfloor t\rfloor}=b\right) d T \\
& +\left(c_{d}-e^{\left.\mu_{d}+\frac{1}{2} \sigma_{d}^{2}\right)} \int_{T_{1}}^{T_{2}} w\left(T_{1}, T_{2}, T\right) P\left(R_{\lfloor T\rfloor}=d \mid R_{\lfloor t\rfloor}=b\right) d T\right. \\
& +\int_{T_{1}}^{T_{2}} w\left(T_{1}, T_{2}, T\right) g_{T} d T .
\end{aligned}
$$


Moreover, if $k$ is such a number that $R_{\lfloor t\rfloor}=i, R_{\lfloor t\rfloor-1} \neq b, \ldots, R_{\lfloor t\rfloor-k+1} \neq$ $b, R_{\lfloor t\rfloor-k}=b$, for $i \in\{s, d\}$ (i.e. the last base regime price before time $t$ was observed $k$-periods earlier), then the forward price is given by:

$$
\begin{aligned}
\left.f_{t \mid\left\{R_{\lfloor t\rfloor}\right.}^{\left[T_{1}, T_{2}\right]}=i, R_{\lfloor t\rfloor-1} \neq b, \ldots, R_{\lfloor t\rfloor-k}=b\right\} & \int_{T_{1}}^{T_{2}} w\left(T_{1}, T_{2}, T\right) P\left(R_{\lfloor T\rfloor}=b \mid R_{\lfloor t\rfloor}=i\right) e^{-\beta(T-\lfloor t\rfloor+k-1)} d T \\
= & X_{\lfloor t\rfloor-k+1, b} \int^{T_{2}} \int_{T_{1}} w\left(T_{1}, T_{2}, T\right) P\left(R_{\lfloor T\rfloor}=b \mid R_{\lfloor t\rfloor}=i\right) \\
& +\int_{\lfloor t\rfloor-k+1} \frac{\alpha}{\beta}\left(1-e^{-\beta(T-u)} \lambda(u) d u\right] d T \\
& +\left(e^{\mu_{s}+\frac{1}{2} \sigma_{s}^{2}}+c_{S}\right) \int_{T_{1}}^{T_{2}} w\left(T_{1}, T_{2}, T\right) P\left(R_{\lfloor T\rfloor}=s \mid R_{\lfloor t\rfloor}=i\right) d T \\
& +\left(c_{d}-e^{\mu_{d}+\frac{1}{2} \sigma_{d}^{2}}\right) \int_{T_{1}}^{T_{2}} w\left(T_{1}, T_{2}, T\right) P\left(R_{\lfloor T\rfloor}=d \mid R_{\lfloor t\rfloor}=i\right) d T \\
& +\int_{T_{1}}^{T_{2}} w\left(T_{1}, T_{2}, T\right) g_{T} d T .
\end{aligned}
$$

Formula (51) is a simple consequence of Eq. (28) and the fact that $R_{\lfloor t\rfloor}=b$ implies that $E^{\lambda}\left(X_{t, b} \mid \mathcal{F}_{t}\right)=X_{t, b}$. In order to show (52), observe that for $R_{\lfloor t\rfloor}=i, R_{\lfloor t\rfloor-1]} \neq$ $b, \ldots, R_{\lfloor t\rfloor-k}=b, i=s, d$, we have

$$
\begin{aligned}
E^{\lambda}\left(X_{t, b} \mid \mathcal{F}_{t}\right)= & E^{\lambda}\left[X_{\lfloor t\rfloor-k+1, b} e^{-\beta(t-\lfloor t\rfloor+k-1)}+\frac{\alpha}{\beta}\left(1-e^{-\beta(t-\lfloor t\rfloor+k-1)}\right)\right. \\
& -\int_{\lfloor t\rfloor-k+1}^{t} e^{-\beta(t-u)} \lambda(u) d u \\
& \left.+\sigma_{b} \int_{\lfloor t\rfloor-k+1}^{t} e^{-\beta(t-u)} d W_{u}^{\lambda} \mid X_{\lfloor t\rfloor-k+1, b]}\right] \\
= & X_{\lfloor t\rfloor-k+1, b} e^{-\beta(t-\lfloor t\rfloor+k-1)}+\frac{\alpha}{\beta}\left(1-e^{-\beta(t-\lfloor t\rfloor+k-1)}\right)
\end{aligned}
$$




$$
-\int_{\lfloor t\rfloor-k+1}^{t} e^{-\beta(t-u)} \lambda(u) d u .
$$

Combining Eqs. (28) and (53) yields the result.

Now we can derive the option price formula. The option price is equal to the expected future payoff. Therefore, we have

$$
C f_{t}^{\left[T_{1}, T_{2}\right]}(K)=e^{-r t} E^{\lambda}\left[\left(f_{t}^{\left[T_{1}, T_{2}\right]}-K\right)^{+} \mid \mathcal{F}_{0}\right]
$$

Observe that the forward price can be written as

$$
\begin{aligned}
f_{t}^{\left[T_{1}, T_{2}\right]}= & \mathbb{I}_{\left\{R_{\lfloor t\rfloor}=b\right\}} f_{t \mid\left\{R_{\lfloor t\rfloor}=b\right\}}^{\left[T_{1}, T_{2}\right]} \\
& +\sum_{i \in\{s, d\}} \sum_{k=1}^{\lfloor t\rfloor} \mathbb{I}_{\left\{R_{\lfloor t\rfloor}=i, R_{\lfloor t\rfloor-1} \neq b, \ldots, R_{\lfloor t\rfloor-k}=b\right\}} f_{t \mid\left\{R_{\lfloor t\rfloor}=i, R_{\lfloor t\rfloor-1} \neq b, \ldots, R_{\lfloor t\rfloor-k}=b\right\}},
\end{aligned}
$$

where $f_{t \mid\left\{R_{\lfloor t\rfloor}\left[T_{1}, T_{2}\right]\right.}$ and $f_{t \mid\left\{R_{\lfloor t\rfloor}=i, R_{\lfloor t\rfloor-1} \neq b, \ldots, R_{\lfloor t\rfloor-k}=b\right\}}^{\left[T_{1}, T_{2}\right]}, i \in\{s, d\}, k=1,2, \ldots,\lfloor t\rfloor$, are given by (51) and (52). Hence,

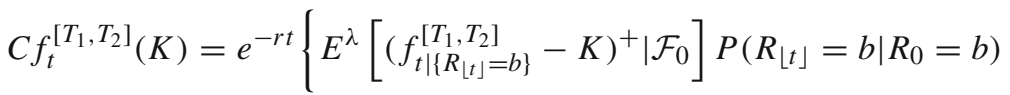

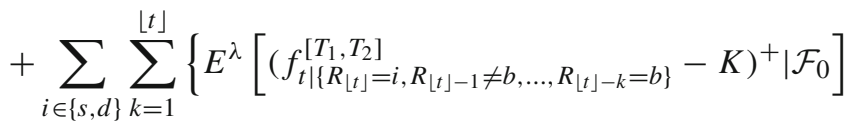

$$
\begin{aligned}
& \left.\times P\left(R_{\lfloor t\rfloor}=i, R_{\lfloor t\rfloor-1} \neq b, \ldots, R_{\lfloor t\rfloor-k}=b \mid R_{0}=b\right)\right\} .
\end{aligned}
$$

Now, observe that

$$
\begin{aligned}
E^{\lambda}\left[\left(f_{t \mid\left\{R_{[t]}\left[T_{1}, T_{2}\right]\right.}-K\right)^{+} \mid \mathcal{F}_{0}\right] & =E^{\lambda}\left[\left(X_{t, b} A_{0}(b)+B_{0}(b)-K\right)^{+} \mid \mathcal{F}_{0}\right] \\
& =A_{0}(b) E^{\lambda}\left[\left(X_{t, b}-\frac{K-B_{0}(b)}{A_{0}(b)}\right)^{+} \mid \mathcal{F}_{0}\right] \\
& =A_{0}(b) C_{t, b}\left(\frac{K-B_{0}(b)}{A_{0}(b)}+g_{t}\right)
\end{aligned}
$$

where $C_{t, b}\left(\frac{K-B_{0}(b)}{A_{0}(b)}+g_{t}\right)$ is the 'base regime part' of the price of a European call option written on the electricity spot price with maturity $t$ and strike $\frac{K-B_{0}(b)}{A_{0}(b)}+g_{t}$, 
see Eq. (21) and $A_{k}, B_{k}$ are defined in Eqs. (30)-(33). Similarly,

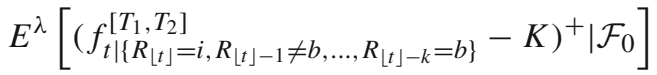

$$
\begin{aligned}
& =A_{k}(i) E^{\lambda}\left[\left(X_{\lfloor t\rfloor-k+1, b}-\frac{K-B_{k}(i)}{A_{k}(i)}\right)^{+} \mid \mathcal{F}_{0}\right] \\
& =A_{k}(i) C_{\lfloor t\rfloor-k+1, b}\left(\frac{K-B_{k}(i)}{A_{k}(i)}+g_{\lfloor t\rfloor-k+1}\right)
\end{aligned}
$$
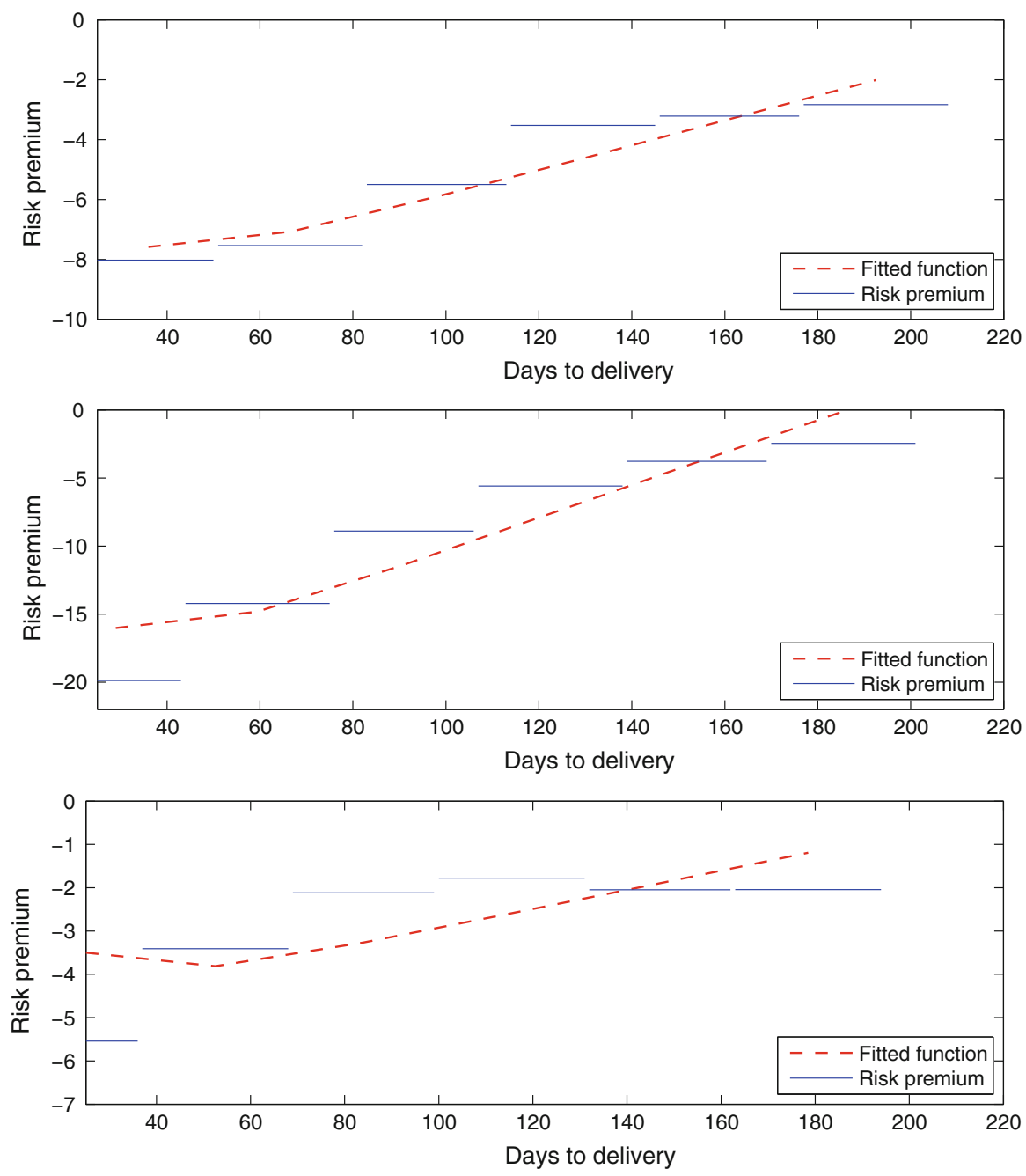

Fig. 6 The risk premium obtained from monthly forward contracts with different delivery periods (blue solid lines) on three consecutive Mondays in January 2011: 10.01.2011 (top panel), 17.01.2011 (middle panel) and 24.01.2011 (bottom panel). Additionally, the fitted function in the case of linear $\lambda(t)$ [see Eq. (36)] is plotted with red dashed lines. (Color figure online) 


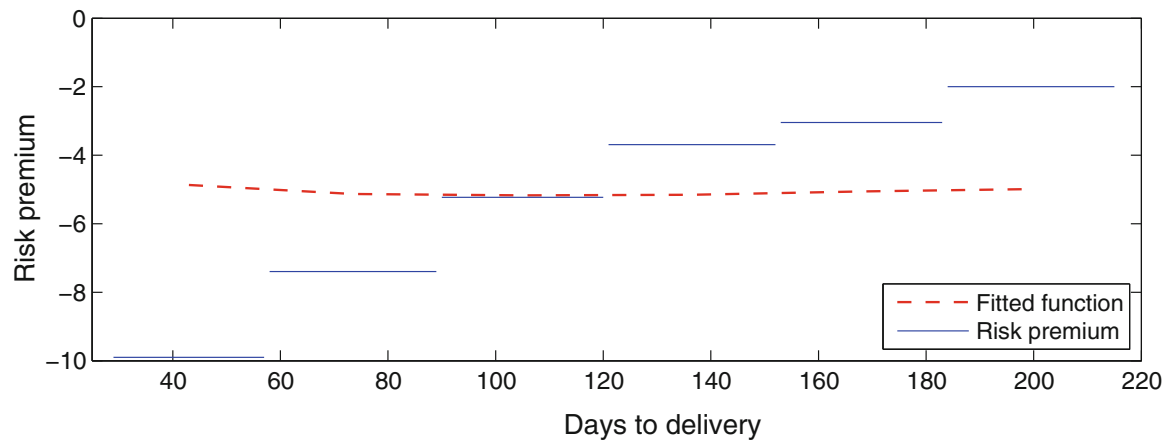

Fig. 7 The risk premium obtained from monthly forward contracts with different delivery periods (blue lines), as well as the fitted function in the case of constant $\lambda$ (see Eq. (36) with $\lambda_{1}=0$ ) plotted with red dashed line. (Color figure online)

for $i \in\{s, d\}$ Finally, combining formulas (54), (57) and (58) completes the proof (Figs. 6, 7).

\section{References}

Becker R, Hurn S, Pavlov V (2007) Modelling spikes in electricity prices. Econ Rec 83:371-382

Benth F, Benth J (2007) The volatility of temperature and pricing of weather derivatives. Quant Financ 7(5):553-561

Benth F, Koekebakker S (2008) Stochastic modeling of financial electricity contracts. Energ Econ 30:11161157

Benth F, Meyer-Brandis T (2009) The information premium in electricity markets. J Energy Markets 2(3):111-140

Benth F, Sgarra C (2012) The risk premium and the esscher transform in power markets. Stoch Anal Appl 30(1):20-43

Benth F, Ekeland L, Hauge R, Nielsen B (2003) A note on arbitrage-free pricing of forward contracts in energy markets. Appl Math Fin 10:325-336

Benth F, Benth J, Koekebakker S (2008a) Stochastic modeling of electricity and related markets. World Scientific, Singapore

Benth F, Cartea A, Kiesel R (2008b) Pricing forward contracts in power markets by the certainty equivalence principle: explaining the sign of the market risk premium. J Bank Financ 32:2006-2021

Bierbrauer M, Menn C (2007) Spot and derivative pricing in the eex power market. J Bank Financ 31:34623485

Bjerksund P, Rasmussen H, Stensland G (2010) Market Energy, natural resources and environmental economics. Springer, chap Valuation and Risk Management in the Norwegian Electricity, pp 167-185. Energy Systems

Bjork T (1997) Interest rate theory. Lect Notes Math 1656:53-122

Cartea A, Figueroa M (2005) Pricing in electricity markets: a mean reverting jump diffusion model with seasonality. Appl Math Financ 12(4):313-335

Clewlow L, Strickland C (1999) Valuing energy options in a one factor model fitted to forward prices. School of Finance and Economics, Technical University of Sydney, Technical report

De Jong C (2006) The nature of power spikes: a regime-switch approach. Stud Nonlinear Dyn E 10(3): Article 3

De Jong C, Huisman R (2002) Option formulas for mean-reverting power prices with spikes. ERIM REPORT SERIES ERS-2002-96-F\&A, Erasmus Research Institute of Management

Dempster A, Laird N, Rubin D (1977) Maximum likelihood from incomplete data via the em algorithm. J R Stat Soc 39:1-38 
Dempster A, Laird N, Rubin D (2013) Fitting semiparametric markov regime-switching models to electricity spot prices. Energ Econ 36:614-624

Deng S (1998) Stochastic models of energy commodity prices and their applications: mean-reversion with jumps and spikes. Working Paper 98-28, PSerc

Erlwein C, Benth F, Mamon R (2010) Hmm filtering and parameter estimation of an electricity spot price model. Energ Econ 32:1034-1043

Ethier R, Mount T (1998) Estimating the volatility of spot prices in restructured electricity markets and the implications for option values. Working Paper 98-31, PSerc

Eydeland A, Wolyniec K (2003) Energy and power risk management. Wiley, New Jersey

Geman H (2005) Commodities and commodity derivatives: pricing and modeling agricultural, metals and energy. Wiley, Chichester

Geman H, Roncoroni A (2006) Understanding the fine structure of electricity prices. J Bus 79:1225-1261

Geman H, Vasciek O (2001) Plugging into electricity. Risk 14:93-97

Girsanov I (1960) On transforming a certain class of stochastic processes by absolutely continuous substitution of measures. Theory Probab Appl 5(3):285-301

Haerdle W, Lopez-Cabrera B (2012) The implied market price of weather risk. Appl Math Financ 19:59-95

Haldrup N, Nielsen M (2006) A regime switching long memory model for electricity prices. J Economet 135(1-2):349-376

Haldrup N, Nielsen F, Nielsen M (2010) A vector autoregressive model for electricity prices subject to long memory and regime switching. Energ Econ 32:1044-1058

Hamilton J (1990) Analysis of time series subject to changes in regime. J Economet 45:39-70

Heath D, Jarrow R, Morton A (1992) Bond pricing and the term structure of interest rates: a new methodology for contingent claims valuation. Econometrica 60(1):77-105

Huisman R (2008) The influence of temperature on spike probability in day-ahead power prices. Energ Econ 30:2697-2704

Huisman R, De Jong C (2003) Option pricing for power prices with spikes. EPRM 7(11):12-16

Huisman R, Mahieu R (2003) Regime jumps in electricity prices. Energ Econ 25:425-434

Hull J, White A (1993) One-factor interest-rate models and the valuation of interest-rate derivative securities. J Financ Quant Anal 28(2):235-254

Janczura J, Weron R (2010) An empirical comparison of alternate regime-switching models for electricity spot prices. Energ Econ 32(5):1059-1073

Janczura J, Weron R (2012) Efficient estimation of markov regime-switching models: an application to electricity wholesale market prices. AStA-Adv Stat Anal 96(3):385-407

Janczura J, Weron R (2013) Goodness-of-fit testing for the marginal distributions of regime-switching models. Adv Stat Anal 97(3):239-270

Kaldor N (1939) Speculation and economic stability. Rev Econ Stud 7:1-27

Kanamura T, Ohashi K (2008) On transition probabilities of regime switching in electricity prices. Energ Econ 30:1158-1172

Karakatsani N, Bunn D (2005) Diurnal reversals in electricity forward premia. Working paper, London Business School

Karakatsani N, Bunn D (2008) Intra-day and regime-switching dynamics in electricity price formation. Energ Econ 30:1776-1797

Kholodnyi V (2005) Modeling power forward prices for power with spikes: a non-markovian approach. Nonlinear Anal 63:958-965

Kim CJ (1994) Dynamic linear models with markov-switching. J Economet 60:1-22

Longstaff F, Wang A (2004) Electricity forward prices: a high-frequency empirical analysis. J Financ 59(4):1877-1900

Lucia J, Schwartz E (2002) Electricity prices and power derivatives: evidence from the nordic power exchange. RDR 5(1):5-50

Lucia J, Torro H (2008) Short-term electricity futures prices: evidence on the time-varying risk premium. Working paper, Dept. of Financial Economics, Universidad de Valencia

Mari C (2008) Random movements of power prices in competitive markets: a hybrid model approach. J Energy Mark 1(2):87-103

Merton R (1976) Option pricing when underlying stock returns are discontinuous. J Financ Econ 3:125-144

Miltersen K (2003) Commodity price modeling that matches current observables: a new approach. Quant Financ 3:51-58 
Mount T, Ning Y, Cai X (2006) Predicting price spikes in electricity markets using a regime-switching model with time-varying parameters. Energ Econ 28:62-80

Musiela M, Rutkowski M (1997) Martingale methods in financial modelling. Springer, Berlin

Nikodym O (1930) Sur une gnralisation des intgrales de m. j. radon. Fund Mathe 15:131-179

Nowotarski J, Tomczyk J, Weron R (2013) Robust estimation and forecasting of the long-term seasonal component of electricity spot prices. Energ Econ 39:13-27

Ronn E, Wimschulte J (2009) Intra-day risk premia in european electricity forward markets. J Energy Mark 2(4):71-96

Vasicek O (1977) An equilibrium characterization of the term structure. J Financ Econ 5:177-188

Weron R (2006) Modeling and forecasting electricity loads and prices: a statistical approach. Wiley, Chichester

Weron R (2008) Market price of risk implied by asian-style electricity options and futures. Energ Econ 30:1098-1115

Weron R (2009) Heavy-tails and regime-switching in electricity prices. Math Method Oper Res 69(3):457473 